\title{
Circuits with a mem-element: invariant manifolds control via pulse programmed sources
}

\author{
Mauro Di Marco • Giacomo Innocenti • \\ Alberto Tesi · Mauro Forti
}

Received: 26 March 2021 / Accepted: 17 August 2021 / Published online: 16 September 2021

(C) The Author(s) 2021

\begin{abstract}
The paper considers the problem of controlling multistability in a general class of circuits composed of a linear time-invariant two-terminal (one port) element, containing linear $R, L, C$ components and ideal operational amplifiers, coupled with one of the mem-elements (memory elements) introduced by Prof. L.O. Chua, i.e., memristors, memcapacitors, and meminductors. First, explicit expressions of the invariant manifolds of the circuit are directly given in terms of the state variables of the two-terminal element and the mem-element. Then, the problem of steering the circuit dynamics from an initial invariant manifold to a final one, and hence to potentially switch among different attractors of the circuit, is addressed by designing pulse shaped control inputs. The control inputs ensure that the transition between the initial and final manifolds is accomplished within a given finite time interval. Moreover, it is shown how the designed control inputs can be implemented by introducing independent voltage and
\end{abstract}

M. Di Marco - M. Forti

Department of Information Engineering and Mathematics, University of Siena, Via Roma 56, 53100 Siena, Italy

e-mail: dimarco@diism.unisi.it

M. Forti

e-mail: forti@diism.unisi.it

G. Innocenti $(\varangle) \cdot$ A. Tesi

Department of Information Engineering, University of

Florence, via S. Marta 3, 50139 Firenze, Italy

e-mail: giacomo.innocenti@unifi.it

A. Tesi

e-mail: alberto.tesi@unifi.it current sources in the two-terminal element. Notably, it turns out that it is always possible to solve the considered control problem by using a unique independent source. Several examples are provided to illustrate how the proposed approach can be applied to different circuits with mem-elements and to highlight the influence of the features of the designed sources on the behavior of the controlled dynamics.

Keywords Mem-element circuits - Multistable dynamics $\cdot$ Multistability control $\cdot$ Pulse programmed sources

\section{Introduction}

The need of an ever growing computing power to handle data-intensive applications is becoming a serious challenge for conventional digital von Neumann computing architectures [1-3]. It is indeed well known that the performance of these architectures is influenced by the physical separation between the central processing unit and the memory chips, which inevitably leads to long latency and energy consumption when addressing data-intensive tasks. In the last years, emerging nanoscale analog devices as memristors and, more generally, mem-elements, have gained a prominent role for overcoming some of the limitations of digital computer architectures by introducing new computing paradigms based on the in-memory and analog computation principles [4-6]. 
The memristor (memory resistor) has been introduced by Prof. Leon Chua in 1971 as the fourth fundamental passive circuit element, while memcapacitors and meminductors are the mem-elements proposed more recently to model memory effects for capacitors and inductors, respectively [7]. The fundamental features of memristors and the other mem-elements are non-volatility, i.e., the capability to hold data in memory without the need of a power supply, and nonlinearity, which makes mem-elements circuits able to generate oscillatory and more complex dynamics $[8,9]$. The combination of these two features makes it possible to devise in-memory computing schemes where the analog dynamics of the mem-element is effectively used for computational purposes and the computation output is stored in the same device [10]. Such a co-location of computation and memory enables low energy consumption and latency reduction. Moreover, in-memory computing allows for high density of the memory arrays, excellent scalability and the capability of $3 \mathrm{D}$ integration, thus making itself as quite a promising novel approach for intensive computing in the area of artificial intelligence and big data [11-16].

This strong interest in mem-elements has stimulated a thorough investigation of the dynamical properties of circuits containing these memory elements. Some of the first investigations of memristor circuits based on analytical and graphical methods can be found in [1721]. Later, it became clear that the state space of circuits containing ideal memristors can be decomposed into a continuum of invariant manifolds (foliation property of the state space), which are indexed by some constant parameter whose value depends on the initial conditions of the circuit. Specifically, in [22,23] a third-order memristor circuit is investigated in the voltage-current domain, while quite general classes of memristor circuits are analyzed in the flux-charge domain (see [24-27] and references therein). In particular, the flux-charge analysis method (FCAM), introduced in [24,25], makes it clear that the rich dynamics displayed by memristor circuits is due to the fact that the state space contains infinitely many invariant manifolds. On each invariant manifold either convergent, or oscillatory, or even more complex behaviors can be displayed, thus implying the coexistence of many different dynamics for a given set of circuit parameters, a property which is referred to as multistability (see, e.g., [9,28-35] and references therein). Also, structural changes in the asymptotic behavior of solutions are observed when varying the initial conditions even for a fixed set of circuit parameters, a peculiar phenomenon referred to as bifurcation without parameters (see, e.g., $[16,25])$. More recently, the flux-charge approach has been extended to much broader classes of circuits containing more than one memristor as well as memcapacitors and meminductors (see, e.g., [8,9,36-41] and references therein). Finally, [42] provides a systematic input-output approach to characterize the dynamical properties of a class of circuits composed of a linear time-invariant two-terminal element coupled with one of the ideal mem-elements. Such an approach can be fruitfully used to predict limit cycles and their bifurcations via the Harmonic balance method (HBM) [4345], as shown in [46,47].

The coexistence of many different attractors makes circuits with mem-elements natural candidates for multistability control, a field of general growing interest (see, e.g., [48] and references therein). Multistability control is indeed considered a fundamental step toward the development of new computational paradigms, such as reservoir computing $[49,50]$. Some contributions to such a problem have been recently given, showing that it is possible to target the memristor circuit dynamics toward the attractor contained in one of the invariant manifolds. In [26], it is shown that multistability can be physically controlled by first obtaining an incremental flux-charge model, which depends on the initial conditions of the dynamic elements of the memristor circuit, and then designing an equivalent circuit realization of the model and tuning the initial conditions via suitable instrumentation. A pulseprogrammed feedforward control law based on FCAM to drive the state on pre-assigned invariant manifolds is proposed in [36] and extended to memristor circuits with nonlinear capacitors and inductors in [51]. Both feedforward and feedback control laws are considered in [52] to drive the state of the memristor MuraliLakshmanan-Chua circuit (see, e.g., [53]) onto a specific invariant manifold within a given time interval. However, the basic issue of how these feedforward and feedback control laws should be implemented in the circuit to physically control multistability remains to be investigated.

This paper aims to address this important practical issue for a fairly general class of circuits with an ideal mem-element. The objective is to investigate if it is possible to steer the circuit dynamics onto a preassigned invariant manifold, within a given time inter- 
Fig. 1 Class of circuits

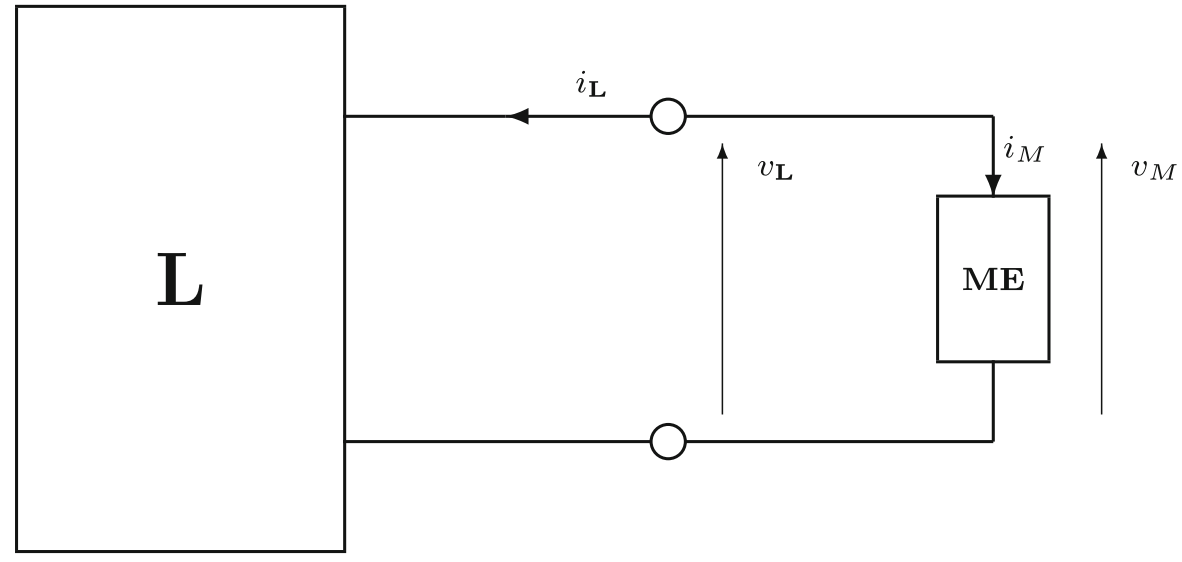

val, by designing suitable pulse programmed feedforward control laws whose circuit implementation can be realized via the introduction of a unique independent source. Preliminary results in this direction have been given in [54,55] for a third-order memristor circuit. In Sect. 2, it is first presented the considered class of circuits which is composed of the interconnection of a linear time-invariant two-terminal (one port) element and a single ideal mem-element. Specifically, the linear two-terminal element can contain linear $R, L$, $C$ components and ideal operational amplifiers, while the mem-element can be a flux- or charge-controlled memristor, a flux- or $\sigma$-controlled capacitor, a $\rho$ - or charge-controlled inductor. Then, on the basis of the approach developed in [42], it is shown that each circuit of the class admits a suitable feedback system representation. Exploiting the structural properties of such a representation, Sect. 3 provides a novel characterization of the invariant manifolds of the circuit without resorting to the flux-charge approach. Specifically, the invariant manifolds are directly expressed in terms of the state variables of the two-terminal element and the mem-element. Section 4 is devoted to the considered control problem of steering the circuit dynamics onto a pre-assigned invariant manifold by introducing in the circuit suitable feedforward pulse shaped control inputs. The issue of how the designed control inputs can be implemented by means of independent voltage and current sources is addressed in Sect. 5. Finally, several examples are presented in Sect. 6 for illustration purposes.

\section{Preliminaries and problem formulation}

Consider the class of circuits depicted in Fig. 1 which is composed of a finite-dimensional causal linear timeinvariant two-terminal (one port) $\mathbf{L}$, with voltage $v_{\mathbf{L}}$ and current $i_{\mathbf{L}}$, and an ideal mem-element $\mathbf{M E}$, with voltage $v_{M}$ and current $i_{M}$, and it does not contain any independent voltage and current sources. Clearly, we have $v_{\mathbf{L}}=v_{M}$ and $i_{\mathbf{L}}=-i_{M}$.

The two-terminal element $\mathbf{L}$, which can be either a passive or an active circuit, is modeled by a finitedimensional causal linear time-invariant dynamical system $\Sigma_{\mathbf{L}}$ with input $u$, output $y$ and state $x$, as depicted in Fig. 2a. Specifically, $\mathbf{L}$ admits two different models according to the choice of the input $u$ and the output $y$ : the current-voltage model $\Sigma_{\mathbf{L}}^{(i)}$, where it is assumed that $u=i_{\mathbf{L}}$ and $y=v_{\mathbf{L}}$, and the voltagecurrent model $\Sigma_{\mathbf{L}}^{(v)}$, where it is assumed that $u=-v_{\mathbf{L}}$ and $y=-i_{\mathbf{L}} \cdot{ }^{1}$ In both cases, the dynamics is assumed to be described by the following state space representation:

$\Sigma_{\mathbf{L}}:\left\{\begin{array}{l}\mathcal{D} x(t)=A x(t)+B u(t) \\ y(t)=C x(t)+D u(t),\end{array}\right.$

where $x \in \mathbb{R}^{n}, u \in \mathbb{R}, y \in \mathbb{R}, A \in \mathbb{R}^{n \times n}, B \in \mathbb{R}^{n \times 1}$, $C \in \mathbb{R}^{1 \times n}, D \in \mathbb{R}^{1 \times 1}$ and $\mathcal{D}$ denotes the differential operator (i.e., $\mathcal{D} f(t)=\dot{f}(t), \mathcal{D}^{2} f(t)=\ddot{f}(t)$, and so on). Clearly, it follows that $u=i_{M}$ and $y=v_{M}$ in the current-voltage model $\Sigma_{\mathbf{L}}^{(i)}$, while $u=-v_{M}$ and $y=i_{M}$ in the voltage-current $\Sigma_{\mathbf{L}}^{(v)}$. We remark that

1 The choice of the minus sign for $v_{\mathbf{L}}$ and $i_{\mathbf{L}}$ in the latter model is useful for putting the circuit of Fig. 1 in the negative feedback representation of Fig. 3a. 


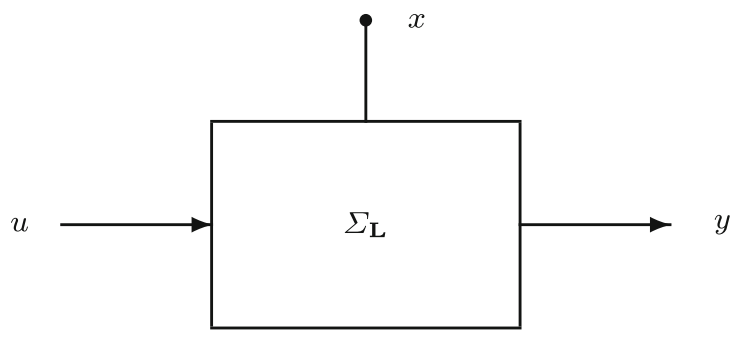

(a)

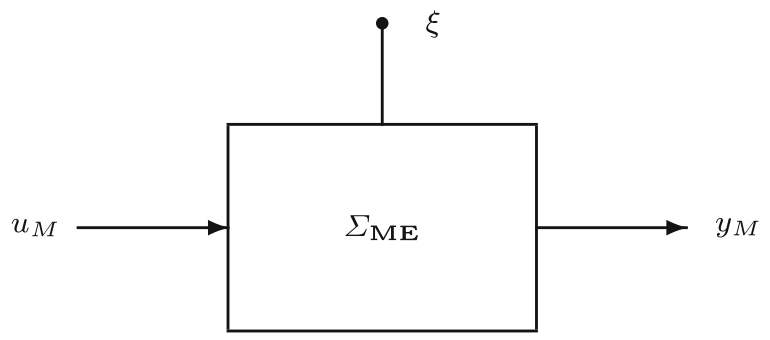

(b)

Fig. 2 a Two-terminal element model $\Sigma_{\mathbf{L}} ; \mathbf{b}$ mem-element model $\Sigma_{\mathbf{M E}}$

$\mathbf{L}$ can be equivalently represented by the input-output relation

$y(t)=L(\mathcal{D}) u(t)$

where $L(\mathcal{D})$ is the following real proper rational function $^{2}$

$L(\mathcal{D})=C\left(\mathcal{D} I_{n}-A\right)^{-1} B+D$.

Consequently, $y(t)$ and $u(t)$ obey the following linear time-invariant ordinary differential equation

$P(\mathcal{D}) y(t)-R(\mathcal{D}) u(t)=0$,

where $P(\mathcal{D})$ and $R(\mathcal{D})$ are the numerator and denominator polynomials of $L(\mathcal{D})$, i.e.,

$L(\mathcal{D})=\frac{R(\mathcal{D})}{P(\mathcal{D})}$

Note that if $s$ is the complex variable, then $L(s)$ is the equivalent impedance of $\mathbf{L}$ in the case of the currentvoltage model $\Sigma_{\mathbf{L}}^{(i)}$ and the equivalent admittance in the case of the voltage-current model $\Sigma_{\mathbf{L}}^{(v)}$. This implies that if the scalar matrix $D$ is zero (i.e., $L(\mathcal{D})$ is strictly proper), then $\mathbf{L}$ can be uniquely described by either the current-voltage model or the voltage-current model, while if $D \neq 0$ (i.e., $L(\mathcal{D})$ is proper but not strictly proper), then both $\Sigma_{\mathbf{L}}^{(i)}$ and $\Sigma_{\mathbf{L}}^{(v)}$ can be equivalently used to model $\mathbf{L}$.

Throughout the paper, the following conditions are enforced on $\mathbf{L}$.

${ }^{2} I_{n}$ denotes the identity matrix of $\operatorname{order} n$.
Assumption 1 The controllability matrix

$\mathcal{R}=\left(B, A B, \ldots, A^{n-1} B\right) \in \mathbb{R}^{n \times n}$

and the observability matrix

$\mathcal{O}=\left(\begin{array}{c}C \\ C A \\ \vdots \\ C A^{n-1}\end{array}\right) \in \mathbb{R}^{n \times n}$

are non-singular.

Remark 1 Assumption 1 ensures that the two-terminal element $\mathbf{L}$ is completely controllable from input $u$ and completely observable from output $y$. Note that the assumption implies that the polynomials $R(\mathcal{D})$ and $P(D)$ in (5) are coprime. Also, under this assumption $\mathbf{L}$ has no loop formed by capacitors only, and no cut-set formed by inductors only.

The mem-element $\mathbf{M E}$ in Fig. 1 can be any of the six mem-elements introduced in $[40,56]$, i.e., a flux-controlled memristor $\left(\mathbf{M R}_{\varphi}\right)$, a charge-controlled memristor $\left(\mathbf{M R}_{q}\right)$, a flux-controlled memcapacitor $\left(\mathbf{M C}_{\varphi}\right)$, a $\sigma$-controlled memcapacitor $\left(\mathbf{M C} \mathbf{C}_{\sigma}\right)$, a chargecontrolled meminductor $\left(\mathbf{M L}_{q}\right)$ and a $\rho$-controlled meminductor $\left(\mathbf{M L}_{\rho}\right)$. Each mem-element can be seen as a finite-dimensional time-invariant nonlinear dynamical system $\Sigma_{\mathbf{M E}}$ with input $u_{M}$, output $y_{M}$ and state $\xi$ as depicted in Fig. 2b. It turns out that $\mathbf{M R}_{\varphi}$ and $\mathbf{M R} \mathbf{R}_{q}$ are modeled by the first-order causal representation

$\Sigma_{\mathbf{M E}}^{(\mathrm{I})}:\left\{\begin{array}{l}\mathcal{D} \xi_{1}(t)=u_{M}(t) \\ y_{M}(t)=\mathcal{D} N\left(\xi_{1}(t)\right)=N^{\prime}\left(\xi_{1}(t)\right) u_{M}(t),\end{array}\right.$ 
Table 1 System $\Sigma_{\text {ME }}$ : state variables, input, output and nonlinear characteristic

\begin{tabular}{lllllll}
\hline $\mathbf{M E}$ & $\mathbf{M R}_{\varphi}$ & $\mathbf{M R}_{q}$ & $\mathbf{M C}_{\varphi}$ & $\mathbf{M L}_{q}$ & $\mathbf{M L}_{\rho}$ & $\mathbf{M C}_{\sigma}$ \\
\hline$\Sigma_{\mathbf{M E}}$ & $\Sigma_{\mathbf{M E}}^{(\mathrm{I})}$ & $\Sigma_{\mathbf{M E}}^{(\mathrm{I})}$ & $\Sigma_{\mathbf{M E}}^{(\mathrm{II})}$ & $\Sigma_{\mathbf{M E}}^{(\mathrm{II})}$ & $\Sigma_{\mathbf{M E}}^{(\mathrm{III})}$ & $\Sigma_{\mathbf{M E}}^{(\mathrm{III})}$ \\
$\xi_{1}$ & $\varphi_{M}$ & $q_{M}$ & $\varphi_{M}$ & $q_{M}$ & $\rho_{M}$ & $\sigma_{M}$ \\
$\xi_{2}$ & & & & $\varphi_{M}$ & $q_{M}$ \\
$u_{M}$ & $v_{M}$ & $i_{M}$ & $v_{M}$ & $i_{M}$ & $v_{M}$ & $i_{M}$ \\
$y_{M}$ & $i_{M}$ & $v_{M}$ & $i_{M}$ & $v_{M}$ & $i_{M}$ & $v_{M}$ \\
$N(\cdot)$ & $q_{M}=N\left(\varphi_{M}\right)$ & $\varphi_{M}=N\left(q_{M}\right)$ & $\sigma_{M}=N\left(\varphi_{M}\right)$ & $\rho_{M}=N\left(q_{M}\right)$ & $q_{M}=N\left(\rho_{M}\right)$ & $\varphi_{M}=N\left(\sigma_{M}\right)$ \\
\hline
\end{tabular}

$\mathbf{M C}_{\varphi}$ and $\mathbf{M L}_{q}$ by the first-order non-causal representation

$$
\Sigma_{\mathbf{M E}}^{(\mathrm{II})}:\left\{\begin{aligned}
\mathcal{D} \xi_{1}(t)= & u_{M}(t) \\
y_{M}(t)= & \mathcal{D}^{2} N\left(\xi_{1}(t)\right)=N^{\prime \prime}\left(\xi_{1}(t)\right) u_{M}^{2}(t) \\
& +N^{\prime}\left(\xi_{1}(t)\right) \mathcal{D}_{M}(t),
\end{aligned}\right.
$$

$\mathbf{M L}_{\rho}$ and $\mathbf{M C}_{\sigma}$ by the second-order causal representation

$$
\Sigma_{\mathbf{M E}}^{(\mathrm{III})}:\left\{\begin{array}{l}
\mathcal{D} \xi_{1}(t)=\xi_{1}(t) \\
\mathcal{D} \xi_{2}(t)=u_{M}(t) \\
y_{M}(t)=\mathcal{D} N\left(\xi_{2}(t)\right)=N^{\prime}\left(\xi_{2}(t)\right) u_{M}(t)
\end{array}\right.
$$

where $\xi_{1}(t), \xi_{2}(t)$ are the state variables, $N(\cdot)$ represents the ME nonlinear characteristic and $N^{\prime}(\cdot), N^{\prime \prime}(\cdot)$ its first- and second-order derivatives. The specific state variables $\xi_{1}, \xi_{2}$, the input $u_{M}$, the output $y_{M}$, and the nonlinear characteristic $N(\cdot)$ pertaining to each $\mathbf{M E}$ are reported in Table 1 in terms of $v_{M}, i_{M}$, the flux $\varphi_{M}$, the charge $q_{M}$, the flux momentum $\rho_{M}$, and the charge momentum $\sigma_{M}$, whose definitions are recalled next:

$$
\begin{aligned}
\varphi_{M}(t) & =\int_{-\infty}^{t} v_{M}(\tau) d \tau=\mathcal{D}^{-1} v_{M}(t) \\
\left(v_{M}(t)\right. & \left.=\mathcal{D} \varphi_{M}(t)\right), \\
q_{M}(t) & =\int_{-\infty}^{t} i_{M}(\tau) d \tau=\mathcal{D}^{-1} i_{M}(t) \\
\left(i_{M}(t)\right. & \left.=\mathcal{D} q_{M}(t)\right), \\
\rho_{M}(t) & =\int_{-\infty}^{t} \varphi_{M}(\tau) d \tau=\mathcal{D}^{-1} \varphi_{M}(t) \\
\left(\varphi_{M}(t)\right. & \left.=\mathcal{D} \rho_{M}(t)\right),
\end{aligned}
$$

$$
\begin{aligned}
\sigma_{M}(t) & =\int_{-\infty}^{t} q_{M}(\tau) d \tau=\mathcal{D}^{-1} q_{M}(t) \\
\left(q_{M}(t)\right. & \left.=\mathcal{D} \sigma_{M}(t)\right) .
\end{aligned}
$$

Throughout the paper, it is assumed that the nonlinear characteristic $N(\cdot)$ vanishes as the argument is equal to zero. It is also required that it is as smooth as needed to ensure existence and uniqueness of the solutions of the system modeling the class of circuits of Fig. 1.

From the above characterization of $\mathbf{L}$ and $\mathbf{M E}$, it follows that the class of circuits of Fig. 1 can be represented via the feedback system of Fig. 3a. Table 1 shows that for each ME the input $u_{M}$ is either the voltage $v_{M}$ or the current $i_{M}$. As a consequence, a unique model $\Sigma_{\mathbf{L}}$ is admissible for each $\mathbf{M E}$, i.e., the one such that $u_{M}=y$. The first row of Table 2 identifies such a relation between $\mathbf{M E}$ and $\mathbf{L}$.

When dealing with feedback systems, an important issue to be considered is its well-posedness, which is strictly related to causality of the interconnected system. It can be shown that well-posedness of the feedback system of Fig. 3a) is ensured once the following additional assumption on $\mathbf{L}$ is enforced.

Assumption 2 Let $\partial P$ and $\partial R$ denote the degree of the denominator and numerator polynomials of $L(\mathcal{D})$. Then, the relative degree of $L(\mathcal{D})$, i.e., $\partial P-\partial R$, is assumed to satisfy:

$$
\begin{aligned}
& \partial P-\partial R>0 \text { if } \Sigma_{\mathbf{M E}}=\Sigma_{\mathbf{M E}}^{(\mathrm{I})} \\
& \partial P-\partial R>1 \text { if } \Sigma_{\mathbf{M E}}=\Sigma_{\mathbf{M E}}^{(\mathrm{II})}
\end{aligned}
$$

Remark 2 From the expression (3) of $L(\mathcal{D})$, it can be verified that condition (15) holds if and only if $D=0$, while condition (16) is satisfied if and only if also $C B=0$. Indeed, this follows by recalling that the 
Table 2 Conditions on $\Sigma_{\mathbf{L}}$ for the feedback system of Fig. 3a

\begin{tabular}{lllllll}
\hline $\mathbf{M E}$ & $\mathbf{M R}_{\varphi}$ & $\mathbf{M R}_{q}$ & $\mathbf{M C}_{\varphi}$ & $\mathbf{M L}_{q}$ & $\mathbf{M L}_{\rho}$ & $\mathbf{M C}_{\sigma}$ \\
\hline$\Sigma_{\mathbf{M E}}$ & $\Sigma_{\mathbf{M E}}^{(\mathrm{I})}$ & $\Sigma_{\mathbf{M E}}^{(\mathrm{I})}$ & $\Sigma_{\mathbf{M E}}^{(\mathrm{II})}$ & $\Sigma_{\mathbf{M E}}^{(\mathrm{II})}$ & $\Sigma_{\mathbf{M E}}^{(\mathrm{III})}$ & $\Sigma_{\mathbf{M E}}^{(\mathrm{III})}$ \\
$\Sigma_{\mathbf{L}}$ & $\Sigma_{\mathbf{L}}^{(i)}$ & $\Sigma_{\mathbf{L}}^{(v)}$ & $\Sigma_{\mathbf{L}}^{(i)}$ & $\Sigma_{\mathbf{L}}^{(v)}$ & $\Sigma_{\mathbf{L}}^{(i)}$ & $\Sigma_{\mathbf{L}}^{(v)}$ \\
$u$ & $i_{\mathbf{L}}$ & $-v_{\mathbf{L}}$ & $i_{\mathbf{L}}$ & $-v_{\mathbf{L}}$ & $i_{\mathbf{L}}$ & $-v_{\mathbf{L}}$ \\
$y$ & $v_{\mathbf{L}}$ & $-i_{\mathbf{L}}$ & $v_{\mathbf{L}}$ & $-i_{\mathbf{L}}$ & $v_{\mathbf{L}}$ & $-i_{\mathbf{L}}$ \\
Matrix & $D=0$ & $D=0$ & $D=0 \& C B=0$ & $D=0 \& C B=0$ & & \\
condition & $D$ & & & & & \\
\hline
\end{tabular}

expression of $L(D)$ in (3) admits the following equivalent representation in series expansion

$$
L(\mathcal{D})=D+\frac{1}{\mathcal{D}} C B+\frac{1}{\mathcal{D}^{2}} C A B+\cdots .
$$

Hence, the additional conditions on the matrices of $\Sigma_{\mathbf{L}}$ to ensure well-posedness are exactly those reported in the last row of Table 2. Note that no conditions are needed when $\Sigma_{\mathbf{M E}}=\Sigma_{\mathbf{M E}}^{\text {(III) }}$, i.e., in the case of a $\sigma$-controlled memcapacitor $\mathbf{M C}_{\sigma}$ and a $\rho$-controlled meminductor $\mathbf{M L}_{\rho}$.

\subsection{Problem formulation}

It is known that the state space of circuits containing one ideal mem-element can be decomposed into a continuum of invariant manifolds (see, e.g., [24-26]). As a consequence, the circuit dynamics can be obtained by collecting the dynamics displayed by a family of reduced-order systems indexed by a constant parameter, whose value determines the specific invariant manifold where the dynamics is confined to lie. Such a constant parameter, which uniquely identifies the corresponding invariant manifold, is hereafter referred to as the manifold index.

Controlling multistability of circuits with memelements, i.e., making the circuit dynamics capable of switching among different attractors, is becoming an issue of increasing interest. In [26], it is shown that multistability can be physically controlled by tuning the initial voltages of the capacitors of the memristor circuit via suitable hardware instrumentation. Some feedforward and feedback control laws have been also proposed for multistability control purposes $[36,51,52]$. However, a systematic procedure for the circuit realization of these control laws has not been given yet.
Figure 4a provides a qualitative illustration of the problem here addressed for circuits of the class of Fig. 1 admitting the feedback system representation of Fig. 3a. Specifically, let $\mathcal{M}_{1}$ and $\mathcal{M}_{2}$ denote the invariant manifolds corresponding to the values $I_{1}$ and $I_{2}$ of the manifold index $I \in \mathbb{R}$. Any trajectory starting on either $\mathcal{M}_{1}$ or $\mathcal{M}_{2}$ remains confined onto the same invariant manifold, eventually converging toward an attractor. Hence, it turns out that a basic issue of multistability control is to design control laws which are capable of steering the circuit dynamics from an invariant manifold to another one in a finite time interval, as qualitatively illustrated in Fig. 4b. Clearly, during this time interval the control laws must change the manifold index from $I_{1}$ to $I_{2}$.

We address this problem by looking at pulse programmed feedforward control laws which can be implemented by introducing independent voltage and current sources in the two-terminal element, as depicted in Fig. 5. To this end, in Sect. 3, we first provide a novel characterization of the invariant manifolds of the feedback system representation of Fig. 3a, which is instrumental for the successive developments. Then, in Sect. 4 we consider the feedback system representation of Fig. 3 b where $\Sigma_{\mathbf{L}}^{(c)}$ is the controlled version of $\Sigma_{\mathbf{L}}$, i.e.,

$\Sigma_{\mathbf{L}}^{(c)}:\left\{\begin{array}{l}\mathcal{D} x(t)=A x(t)+B u(t)+F w(t) \\ y(t)=C x(t)+D u(t)\end{array}\right.$

with $w \in \mathbb{R}$ being the feedforward control input and $F \in \mathbb{R}^{1 \times n}$ the vector defining how the control input affects the two-terminal element $\mathbf{L}$.

The problem under investigation can be formally stated as follows. Let $I \in \mathbb{R}$ denote the manifold index and $\mathcal{M}_{1}, \mathcal{M}_{2}$ be the invariant manifolds corresponding to $I=I_{1}, I=I_{2}$, respectively. Suppose that for all $t \in$ 


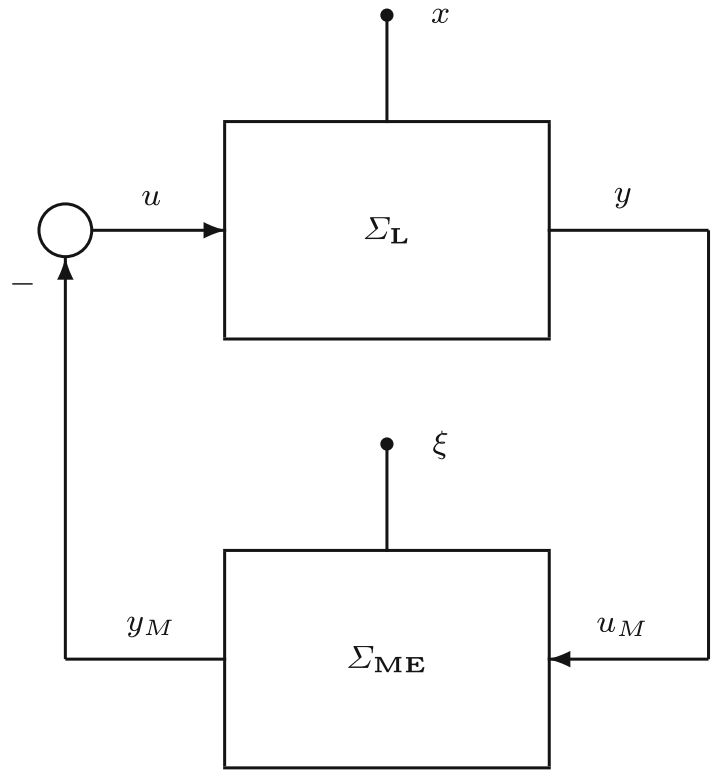

(a)

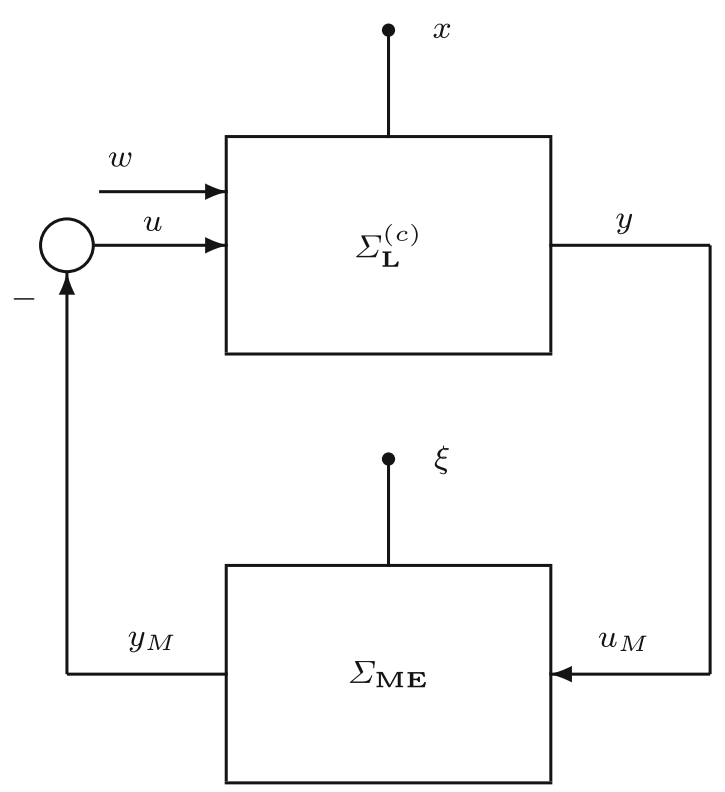

(b)

Fig. 3 a Feedback system representation of the class of circuits of Fig. 1; b controlled version of the feedback system representation of the class of circuits of Fig. 1

$\left[t_{0}, t_{1}\right], t_{0}<t_{1}$, the dynamics lies onto $\mathcal{M}_{1}$. Then, the objective is to design $F$ in order to steer the dynamics from $\mathcal{M}_{1}$ to $\mathcal{M}_{2}$ within the time interval $\left[t_{1}, t_{2}\right], t_{1}<$ $t_{2}<+\infty$, for any input $w$ with the following pulse shape:

$w(t)=\left\{\begin{array}{lll}0 & \text { if } t \in\left[t_{0}, t_{1}\right] \\ \bar{w}(t) & \text { if } t \in\left(t_{1}, t_{2}\right) \\ 0 & \text { if } t \in\left[t_{2},+\infty\right)\end{array}\right.$

where $\bar{w}(t)$ is any piecewise-continuous signal in $\left[t_{1}, t_{2}\right]$ satisfying the following area constraint:

$\int_{t_{1}}^{t_{2}} \bar{w}(t) d t=I_{2}-I_{1}$

It is worth noting that the shape of the pulses satisfying (20) is expected to affect the transient behavior when the circuit dynamics moves from $\mathcal{M}_{1}$ toward $\mathcal{M}_{2}$ and hence between the corresponding attractors.

The final step concerns the implementation of the obtained $F$ and $w$ within the two-terminal element, i.e., to determine where the sources $V_{1}^{(s)}, \ldots, V_{i}^{(s)}, \ldots$ and/or current $I_{1}^{(s)}, \ldots, I_{i}^{(s)}, \ldots$ should be located inside $\mathbf{L}$ (see Fig. 5). This issue is addressed in Sect. 5.

\section{Invariant manifolds characterization}

In this section, a novel analytical characterization of the invariant manifolds of any circuit of the class of Fig. 1 is provided in terms of the state variable $x$ and $\xi$ of $\Sigma_{\mathbf{L}}$ and $\Sigma_{\mathbf{M E}}$, respectively. In particular, the invariant manifolds are parameterized via a scalar quantity $I \in \mathbb{R}$, which is referred to as the manifold index. Such a parameterization is developed by exploiting the structural properties of the feedback representation in Fig. 3a of the circuit of Fig. 1. The next result plays a key role in the sought characterization.

Lemma 1 Let Assumption 1 hold and consider the system of $n+1$ linear equations

$\left\{\begin{array}{l}v A+\lambda C=0 \\ v B-\mu=0\end{array}\right.$

where $v^{\top} \in \mathbb{R}^{n}, \lambda \in \mathbb{R}, \mu \in \mathbb{R}$. Then, the solution set of (21), i.e.,

$$
\mathcal{S}=\left\{(v, \lambda, \mu)^{\top} \in \mathbb{R}^{n+2}: v A+\lambda C=0, v B-\mu=0\right\}
$$




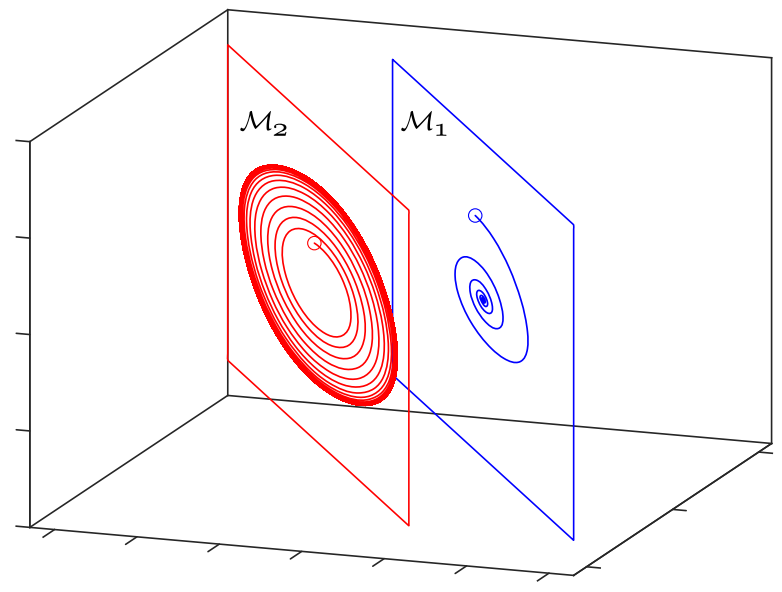

(a)

Fig. 4 a The invariant manifolds $\mathcal{M}_{1}$ of index $I=I_{1}$ and $\mathcal{M}_{2}$ of index $I=I_{2}$ are depicted in blue and red, respectively. The trajectories lying on $\mathcal{M}_{1}$ and $\mathcal{M}_{2}$ converge toward an equilibrium point and a limit cycle, respectively. The initial conditions are marked with $\circ$. b The trajectory starts from the initial conditions on $\mathcal{M}_{1}$ marked with $\circ$ at $t=t_{0}$ and it remains on $\mathcal{M}_{1}$ for $t \in\left[t_{0}, t_{1}\right], t_{1}>t_{0}$ (blue trajectory). At $t=t_{1}$ the control

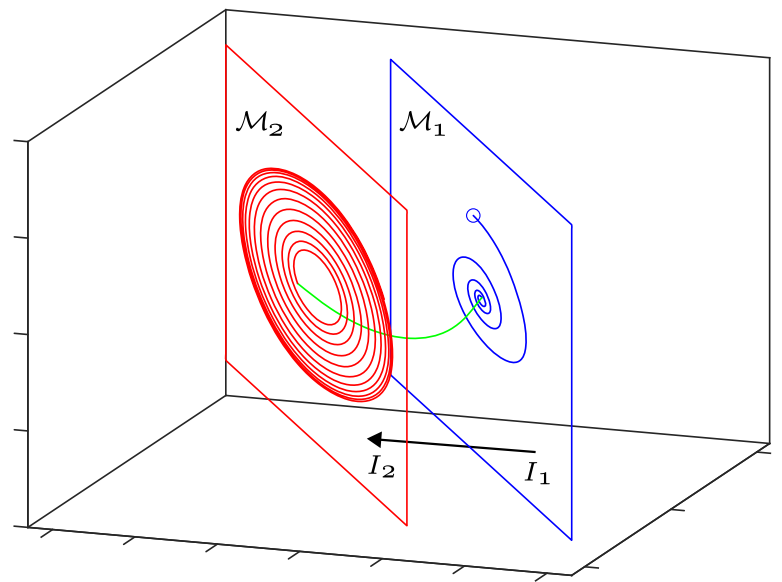

(b)

law is activated and the trajectory moves away from $\mathcal{M}_{1}$ (green trajectory) reaching $\mathcal{M}_{2}$ at $t=t_{2}, t_{2}>t_{1}$, when the control law is turned off. Then, for $t>t_{2}$ the trajectory remains on $\mathcal{M}_{2}$ and converges to the limit cycle (red trajectory). The control law is designed in order to make the manifold index $I$ varying from $I_{1}$ to $I_{2}$ in the time interval $\left[t_{1}, t_{2}\right]$. (Color figure online)

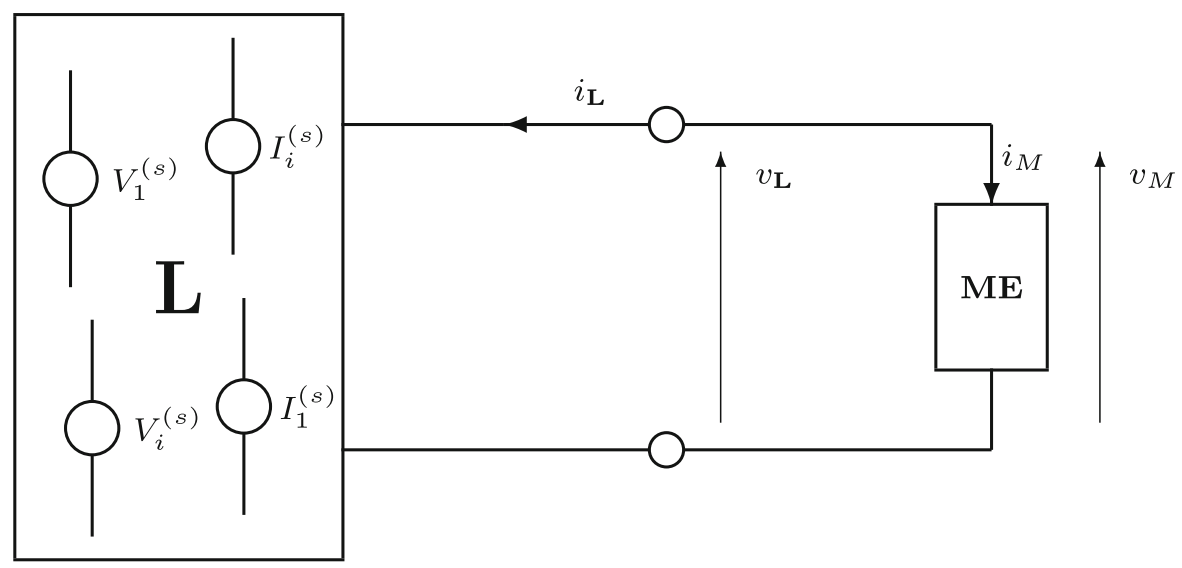

Fig. 5 Controlled class of circuits: independent voltage $V_{1}^{(s)}, \ldots, V_{i}^{(s)}, \ldots$ and/or current $I_{1}^{(s)}, \ldots, I_{i}^{(s)}, \ldots$ sources are introduced in the two-terminal element $\mathbf{L}$

is a one-dimensional linear subspace of $\mathbb{R}^{n+2}$ such that

$\mathcal{S}=\operatorname{span}\left\{\left(-C A^{-1}, 1,-C A^{-1} B\right)^{\top}\right\}$

if $A$ is non-singular, and by

$\mathcal{S}=\operatorname{span}\left\{(\bar{v}, 0, \bar{v} B)^{\top}\right\}$ with $\bar{v}^{\top} \in \mathbb{R}^{n+2}, \bar{v} \neq 0_{1 \times n}$, such that $\bar{v} A=0$, if $A$ is singular.

Proof See the appendix.

Remark 3 Note that $A$ is non-singular whenever $\mathbf{L}$ is passive. In fact, if $L$ is passive, then its impedance/ admittance $L(s)$ must be positive real and hence the denominator $P(s)$ of $L(s)$ must be a Hurwitz poly- 
nomial, which in turn implies that $P(0) \neq 0$. Since $P(s)=\operatorname{det}\left(s I_{n}-A\right)$, this last condition is indeed equivalent to $\operatorname{det}(A) \neq 0$.

Let us first consider the system of Fig. 3a with $\Sigma_{\mathbf{M E}}=$ $\Sigma_{\mathbf{M E}}^{(\mathrm{I})}$, i.e., the case of $\mathbf{M} \mathbf{R}_{\varphi}$ and $\mathbf{M} \mathbf{R}_{q}$. By exploiting (1), (8) and Assumption 2, it can be verified that in this case the system of Fig. 3a obeys the following equations:

$$
\Sigma:\left(\begin{array}{c}
\mathcal{D} x(t) \\
\mathcal{D} \eta(t)
\end{array}\right)=\left(\begin{array}{cc}
A & 0_{n \times 1} \\
C & 0
\end{array}\right)\left(\begin{array}{c}
x(t) \\
\eta(t)
\end{array}\right)-\left(\begin{array}{c}
B \\
0
\end{array}\right) \mathcal{D} r(t)
$$

once $\eta(t) \doteq \xi_{1}(t)$ and $r(t) \doteq N\left(\xi_{1}(t)\right)$. Note that $\Sigma$ is a linear time-invariant system with $\left(x^{\top}, \eta\right)^{\top} \in \mathbb{R}^{n+1}$ as state vector and $\mathcal{D} r(t)$ as input. Exploiting this special structure, the following result is derived:

Proposition 1 Let Assumptions 1 and 2 hold. Then, the invariant manifolds $\mathcal{M}_{I}, I \in \mathbb{R}$, of the feedback system of Fig. $3 a$ in the case $\Sigma_{\mathbf{M E}}=\Sigma_{\mathbf{M E}}^{(\mathrm{I})}$ are given by

$$
\begin{aligned}
\mathcal{M}_{I}= & \left\{x \in \mathbf{R}^{n}, \xi_{1} \in \mathbb{R}:\right. \\
& \left.\xi_{1}-C A^{-1} x-C A^{-1} B N\left(\xi_{1}\right)=I\right\},
\end{aligned}
$$

if $A$ is non-singular, and by

$\mathcal{M}_{I}=\left\{x \in \mathbf{R}^{n}, \xi_{1} \in \mathbb{R}: \bar{v} x+\bar{v} B N\left(\xi_{1}\right)=I\right\}$

with $\bar{v} \neq 0$, such that $\bar{v} A \neq 0$, if $A$ is singular. In both cases, the following set equivalence

$\left\{x \in \mathbb{R}^{n}, \xi_{1} \in \mathbb{R}:\left(x^{\top}, \xi_{1}\right)^{\top} \in \bigcup_{I \in \mathbb{R}} \mathcal{M}_{I}\right\} \equiv \mathbb{R}^{n+1}$

holds, i.e., the state space of the feedback system is decomposed in a continuum of invariant manifolds.

Proof We first observe that if

$\mathcal{L}_{I}=\left\{x \in \mathbf{R}^{n}, \eta \in \mathbb{R}: v x+\lambda \eta+\mu r=I\right\}$

is an invariant linear manifold of $\Sigma$ then, since $\eta=\xi_{1}$ and $r=N\left(\xi_{1}\right)$,

$$
\mathcal{M}_{I}=\left\{x \in \mathbf{R}^{n}, \xi_{1} \in \mathbb{R}: v x+\lambda \xi_{1}+\mu N\left(\xi_{1}\right)=I\right\}
$$

is an invariant manifold of the feedback system of Fig. 3a. Now, (29) is an invariant manifold for each $I \in \mathbb{R}$ if and only if

$v \mathcal{D} x(t)+\lambda \mathcal{D} \eta(t)+\mu \mathcal{D} r(t)=0$

and hence, taking into account the expressions of $\mathcal{D} x(t)$ and $\mathcal{D} \eta(t)$ in (25), if and only if

$(v A+\lambda) x(t)+(\mu-v B) \mathcal{D} r(t)=0$

for all $x(t) \in \mathbb{R}^{n}, r(t) \in \mathbb{R}$. According to Lemma 1 , condition (32) is satisfied if and only if $(v, \lambda, \mu)^{\top} \in \mathcal{S}$. Hence, the proof of (26)-(27) readily follows from (30) and Lemma 1. Finally, to prove (28), it is enough to observe that

$\left\{x \in \mathbb{R}^{n}, \eta \in \mathbb{R}:\left(x^{\top}, \eta\right)^{\top} \in \bigcup_{I \in \mathbb{R}} \mathcal{L}_{I}\right\} \equiv \mathbb{R}^{n+1}$

and

$\bigcup_{I \in \mathbb{R}} \mathcal{L}_{I} \equiv \bigcup_{I \in \mathbb{R}} \mathcal{M}_{I}$

once $\eta$ and $r$ are replaced by $\xi_{1}$ and $N\left(\xi_{1}\right)$, respectively.

Remark 4 The expression of $\mathcal{M}_{I}$ implies that, in the case $\Sigma_{\mathbf{M E}}=\Sigma_{\mathbf{M E}}^{(\mathrm{I})}$, each trajectory of the feedback system of Fig. 3a with initial conditions $x\left(t_{0}\right), \xi_{1}\left(t_{0}\right)$ satisfying either

$\xi_{1}\left(t_{0}\right)-C A^{-1} x\left(t_{0}\right)-C A^{-1} B N\left(\xi_{1}\left(t_{0}\right)\right)=I$,

if $A$ is non-singular, or

$\bar{v} x\left(t_{0}\right)+\bar{v} B N\left(\xi_{1}\left(t_{0}\right)\right)=I$,

if $A$ is singular, lies on $\mathcal{M}_{I}$ for all $t \geq t_{0}$. This implies that the value of the index manifold $I$ depends on the initial conditions of the circuit.

Remark 5 From the proof of Proposition 1, it follows that system $\Sigma$ admits linear invariant subspaces of the form $v x+\lambda \eta+\mu r=I$ (see (29)). This implies that $\Sigma$ cannot be completely controllable from the input $r$. Indeed, by introducing the new state vector $\bar{x}=x-B r$, $\Sigma$ can be rewritten equivalently as 
$\Sigma_{e q}:\left(\begin{array}{c}\mathcal{D} \bar{x}(t) \\ \mathcal{D} \eta(t)\end{array}\right)=\left(\begin{array}{cc}A & 0_{n \times 1} \\ C & 0\end{array}\right)\left(\begin{array}{c}\bar{x}(t) \\ \eta(t)\end{array}\right)-\left(\begin{array}{c}A B \\ C B\end{array}\right) r(t)$.

Now, applying the PBH test (see, e.g., [57]) to the zero eigenvalue of the state matrix, we get

$\operatorname{rank}\left(\begin{array}{ccc}A & 0_{n \times 1}-A B \\ C & 0 & -C B\end{array}\right)=\operatorname{rank}\left(\begin{array}{c}A \\ C\end{array}\right)=n<n+1$,

which shows that $\Sigma_{e q}$, and hence $\Sigma$, is not completely controllable from the input $r$.

We now consider the case when $\Sigma_{\mathbf{M E}}=\Sigma_{\mathbf{M E}}^{(\text {II) }}$, i.e., the case of $\mathbf{M C}_{\varphi}$ and $\mathbf{M} \mathbf{L}_{q}$. From (1), (9) and Assumption 2, it follows that the system of Fig. 3a is described by system $\Sigma$ in (25) once $\eta(t) \doteq \xi_{1}(t)$ and $r(t) \doteq$ $\mathcal{D} N\left(\xi_{1}(t)\right)$. Hence, the next result holds true.

Proposition 2 Let Assumptions 1 and 2 hold. Then, the invariant manifolds $\mathcal{M}_{I}, I \in \mathbb{R}$, of the feedback system of Fig. $3 a$ in the case $\Sigma_{\mathbf{M E}}=\Sigma_{\mathbf{M E}}^{(\mathrm{II})}$ are given by

$$
\begin{aligned}
\mathcal{M}_{I}= & \left\{x \in \mathbf{R}^{n}, \xi_{1} \in \mathbb{R}:\right. \\
& \left.\xi_{1}-C A^{-1} x-C A^{-1} B N^{\prime}\left(\xi_{1}\right)=I\right\},
\end{aligned}
$$

if $A$ is non-singular, and by

$\mathcal{M}_{I}=\left\{x \in \mathbf{R}^{n}, \xi_{1} \in \mathbb{R}: \bar{v} x+\bar{v} B N^{\prime}\left(\xi_{1}\right)=I\right\}$,

with $\bar{v} \neq 0$, such that $\bar{v} A \neq 0$, if $A$ is singular. In both cases, the following set equivalence

$\left\{x \in \mathbb{R}^{n}, \xi_{1} \in \mathbb{R}:\left(x^{\top}, \xi_{1}\right)^{\top} \in \bigcup_{I \in \mathbb{R}} \mathcal{M}_{I}\right\} \equiv \mathbb{R}^{n+1}$

holds.

Proof The proof parallels that of Proposition 1 with the unique difference that now $r=\mathcal{D} N\left(\xi_{1}\right)$.

Let us finally consider the system of Fig. 3a with $\Sigma_{\mathbf{M E}}=\Sigma_{\mathbf{M E}}^{(\mathrm{III})}$, i.e., the case of $\mathbf{M C _ { \sigma }}$ and $\mathbf{M L}_{\rho}$. In this case, we need to extend the system $\Sigma$ in (25) as follows:

$$
\begin{aligned}
\Sigma^{(e)}: & \left(\begin{array}{l}
\mathcal{D} x(t) \\
\mathcal{D} \eta(t) \\
\mathcal{D} \zeta(t)
\end{array}\right)=\left(\begin{array}{ccc}
A & 0_{n \times 1} & 0_{n \times 1} \\
C & 0 & 0 \\
0 & 1 & 0
\end{array}\right)\left(\begin{array}{c}
x(t) \\
\eta(t) \\
\zeta(t)
\end{array}\right) \\
& -\left(\begin{array}{l}
B \\
0 \\
0
\end{array}\right) \mathcal{D} r(t),
\end{aligned}
$$

where the additional state variable $\zeta$ is the time-integral of $\eta$. By exploiting (1), (10) and Assumption 2, it can be readily verified that $\Sigma^{(e)}$ describes the dynamics of the system of Fig. $3 \mathrm{a}$ in the case $\Sigma_{\mathbf{M E}}=\Sigma_{\mathbf{M E}}^{(\mathrm{III})}$ once $\zeta(t) \doteq \xi_{1}(t), \eta(t) \doteq \xi_{2}(t)$ and $r(t) \doteq N\left(\xi_{2}(t)\right)$. Note that $\Sigma^{(e)}$ is a linear time-invariant system with $\left(x^{\top}, \eta, \zeta\right)^{\top} \in \mathbb{R}^{n+2}$ as state vector and $\mathcal{D} r(t)$ as input. The next result holds true.

Proposition 3 Let Assumptions 1 and 2 hold. Then, the invariant manifolds $\mathcal{M}_{I}, I \in \mathbb{R}$, of the feedback system of Fig. $3 a$ in the case $\Sigma_{\mathbf{M E}}=\Sigma_{\mathbf{M E}}^{(\mathrm{III})}$ are given by

$$
\begin{aligned}
\mathcal{M}_{I}= & \left\{x \in \mathbf{R}^{n}, \xi_{1} \in \mathbb{R}, \xi_{2} \in \mathbb{R}:\right. \\
& \left.\xi_{1}-C A^{-1} x-C A^{-1} B N\left(\xi_{2}\right)=I\right\},
\end{aligned}
$$

if $A$ is non-singular, and by

$\mathcal{M}_{I}=\left\{x \in \mathbf{R}^{n}, \xi_{1} \in \mathbb{R}, \xi_{2} \in \mathbb{R}: \bar{v} x+\bar{v} B N\left(\xi_{2}\right)=I\right\}$,

with $\bar{v} \neq 0$, such that $\bar{v} A \neq 0$, if $A$ is singular. In both cases, the following set equivalence

$$
\left\{x \in \mathbb{R}^{n}, \xi_{1} \in \mathbb{R}:\left(x^{\top}, \xi_{1}, \xi_{2}\right)^{\top} \in \bigcup_{I \in \mathbb{R}} \mathcal{M}_{I}\right\} \equiv \mathbb{R}^{n+2}
$$

holds.

Proof The proof parallels that of Proposition 1. First, observe that

$$
\begin{array}{r}
\mathcal{L}_{I}^{(e)}=\left\{x \in \mathbf{R}^{n}, \eta \in \mathbb{R}, \zeta \in \mathbb{R}:\right. \\
v x+\lambda \eta+v \zeta+\mu r=I\}
\end{array}
$$


Table 3 Explicit expressions of the invariant manifolds $\mathcal{M}_{I}$ for each ME: $I \in \mathbb{R}$ is the manifold index; $\bar{v} \neq 0_{1 \times n}$ is a fixed vector such that $\bar{v} A=0 ; N(\cdot)$ is the ME nonlinear characteristic and $N^{\prime}(\cdot)$ its derivative (see Table 1)

\begin{tabular}{lll}
\hline $\mathbf{M E}$ & $\mathcal{M}_{I}-A$ non-singular & $\mathcal{M}_{I}-A$ singular \\
\hline $\mathbf{M R}_{\varphi}$ & $\varphi_{M}-C A^{-1} x-C A^{-1} B N\left(\varphi_{M}\right)=I$ & $\bar{v} x+\bar{v} B N\left(\varphi_{M}\right)=I$ \\
$\mathbf{M R}_{q}$ & $q_{M}-C A^{-1} x-C A^{-1} B N\left(q_{M}\right)=I$ & $\bar{v} x+\bar{v} B N\left(q_{M}\right)=I$ \\
$\mathbf{M C}_{\varphi}$ & $\varphi_{M}-C A^{-1} x-C A^{-1} B N^{\prime}\left(\varphi_{M}\right) C x=I$ & $\bar{v} x+\bar{v} B N\left(\varphi_{M}\right)^{\prime}\left(\varphi_{M}\right) C x=I$ \\
$\mathbf{M L}_{q}$ & $q_{M}-C A^{-1} x-C A^{-1} B N^{\prime}\left(q_{M}\right) C x=I$ & $\bar{v} x+\bar{v} B N^{\prime}\left(q_{M}\right) C x=I$ \\
$\mathbf{M L}_{\rho}$ & $\varphi_{M}-C A^{-1} x-C A^{-1} B N\left(\rho_{M}\right)=I$ & $\bar{v} x+\bar{v} B N\left(\rho_{M}\right)=I$ \\
$\mathbf{M C}_{\sigma}$ & $q_{M}-C A^{-1} x-C A^{-1} B N\left(\sigma_{M}\right)=I$ & $\bar{v} x+\bar{v} B N\left(\sigma_{M}\right)=I$ \\
\hline
\end{tabular}

is an invariant manifold of $\Sigma^{(e)}$ for each $I \in \mathbb{R}$ if and only if

$v \mathcal{D} x(t)+\lambda \mathcal{D} \eta(t)+v \mathcal{D} \zeta(t)+\mu \mathcal{D} r(t)=0$

and hence if and only if

$(v A+\lambda) x(t)+v \eta(t)+(\mu-v B) \mathcal{D} r(t)=0$

for all $x(t) \in \mathbb{R}^{n}, \eta(t) \in \mathbb{R}, r(t) \in \mathbb{R}$. Then, $\mathcal{L}_{I}^{(e)}$ is an invariant manifold of $\Sigma^{(e)}$ if and only if $(v, \lambda, \mu)^{\top} \in \mathcal{S}$ and $v=0$ and hence the proof is completed by replacing, $\eta, \zeta$ and $r$ with $\xi_{1}, \xi_{2}$ and $N\left(\xi_{2}\right)$, respectively.

Remark 6 It is worth noting that Remark 4 also applies to the cases when $\Sigma_{\mathbf{M E}}=\Sigma_{\mathbf{M E}}^{(\mathrm{II})}$ and $\Sigma_{\mathbf{M E}}=\Sigma_{\mathbf{M E}}^{(\mathrm{II})}$. Moreover, we note that also $\Sigma^{(e)}$ is not completely controllable from the input $r$, since it contains $\Sigma$ as a subsystem.

The explicit expressions of the invariant manifolds pertaining to each ME when $A$ is either singular or nonsingular are readily obtained exploiting Table 1 and they are summarized in Table 3. Note that the invariant manifolds are directly expressed in terms of the state vector $x$ of the two-terminal element $\mathbf{L}$ and the state vector $\xi$ of the mem-element ME (the specific expressions of $\xi_{1}, \xi_{2}$ and the nonlinear characteristic pertaining to each $\mathbf{M E}$ are reported in the second, third and last rows of Table 1).

\section{Design of pulse shaped feedforward control inputs}

In this section, we consider the controlled system of Fig. $3 \mathrm{~b}$ where $\Sigma_{L}^{(c)}$ is given in (18). We are interested in characterizing the control vectors $F$ capable to solve the problem described in Subsection 2.1, i.e., to steer the circuit dynamics from one manifold to another one within a given time interval, with $w(t)$ having the pulse shape (19) and satisfying the area constraint (20).

Clearly, for any time $t \in\left[t_{0}, t_{1}\right)$ and $t \in\left[t_{2},+\infty\right)$, the controlled system of Fig. $3 b$ displays the same dynamics of the uncontrolled one of Fig. 3a. Hence, the state vectors $x(t)$ and $\xi(t)$ are confined to lie onto some initial manifold $\mathcal{M}_{1}$ for $t \in\left[t_{0}, t_{1}\right)$ and onto some final manifold $\mathcal{M}_{2}$ for $t \in\left[t_{2},+\infty\right)$. Let us denote by $I_{1}$ and $I_{2}$ the values of the index $I$ corresponding to the initial and final manifolds, respectively. Hence, according to Proposition 1, in the case of either $\mathbf{M R}_{\varphi}$ or $\mathbf{M} \mathbf{R}_{q}$ as ME we have $\mathcal{M}_{1} \equiv \mathcal{M}_{I_{1}}, \mathcal{M}_{2} \equiv \mathcal{M}_{I_{2}}$ and the state vector $\left(x^{\top}, \xi_{1}\right)^{\top} \in \mathbb{R}^{n+1}$ satisfies either

$\left\{\begin{array}{l}\xi_{1}(t)-C A^{-1} x(t)-C A^{-1} B N\left(\xi_{1}(t)\right)=I_{1} \\ \forall t \in\left[t_{0}, t_{1}\right] \\ \xi_{1}(t)-C A^{-1} x(t)-C A^{-1} B N\left(\xi_{1}(t)\right)=I_{2} \\ \forall t \in\left[t_{2},+\infty\right)\end{array}\right.$,

if $A$ is non-singular, or

$$
\begin{cases}\bar{v} x(t)+\bar{v} B N\left(\xi_{1}(t)\right)=I_{1} & \forall t \in\left[t_{0}, t_{1}\right] \\ \bar{v} x(t)+\bar{v} B N\left(\xi_{1}(t)\right)=I_{2} & \forall t \in\left[t_{2},+\infty\right)\end{cases}
$$

if $A$ is singular. Clearly, according to Propositions 2 and 3 , analogous expressions can be readily derived in case of $\mathbf{M C}_{\varphi}, \mathbf{M L}_{q}, \mathbf{M L} \mathbf{L}_{\rho}$ and $\mathbf{M \mathbf { C } _ { \sigma }}$. The following characterization of the feedforward control laws ensuring to steer the dynamics from $\mathcal{M}_{1}$ to $\mathcal{M}_{2}$ is obtained.

Proposition 4 Let $\mathcal{M}_{1}$ and $\mathcal{M}_{2}$ be the initial and final invariant manifolds, let $I_{1}$ and $I_{2}$ be the corresponding 
values of the manifold index I, respectively, and let $w$ be any control input having the pulse shape (19) and satisfying condition (20). Then, the system dynamics is driven from $\mathcal{M}_{1}$ to $\mathcal{M}_{2}$ within the time interval $\left[t_{1}, t_{2}\right]$ if and only if $F \in \mathbb{R}^{n}$ satisfies either

$C A^{-1} F=-1$,

\section{if $A$ is non-singular, or}

$\bar{v} F=-1$,

\section{if $A$ is singular.}

Proof Let us consider the case of $\Sigma_{\mathbf{M E}}=\Sigma_{\mathbf{M E}}^{(\mathrm{I})}$, i.e., $\mathbf{M E}$ is either $\mathbf{M R}_{\varphi}$ or $\mathbf{M} \mathbf{R}_{q}$ and suppose first that $A$ is non-singular. Since in this case $\mathcal{M}_{1} \equiv \mathcal{M}_{I_{1}}^{(\mathrm{I})}$ and $\mathcal{M}_{2} \equiv \mathcal{M}_{I_{2}}^{(\mathrm{I})}$, by introducing the scalar variable

$z \doteq \xi_{1}-C A^{-1} x-C A^{-1} B N\left(\xi_{1}\right)$

and according to (26), $\mathcal{M}_{1}$ and $\mathcal{M}_{2}$ can be expressed as

$\mathcal{M}_{1}=\left\{x \in \mathbb{R}^{n}, \xi_{1} \in \mathbb{R}: \quad z=I_{1}\right\}$,

and

$\mathcal{M}_{2}=\left\{x \in \mathbb{R}^{n}, \xi_{1} \in \mathbb{R}: \quad z=I_{2}\right\}$,

respectively. Clearly, from (49) and (53) it follows that $\left(x^{\top}(t), \xi_{1}(t)\right)^{\top} \in \mathcal{M}_{1}$ if and only if $z(t)=I_{1}$ and $\left(x^{\top}(t), \xi_{1}(t)\right)^{\top} \in \mathcal{M}_{2}$ if and only if $z(t)=I_{2}$. Moreover, exploiting (8) and (18), the time-derivative of $z(t)$ can be computed as

$$
\begin{aligned}
\mathcal{D} z(t)= & \mathcal{D} \xi_{1}-C A^{-1} \mathcal{D} x(t)-C A^{-1} B \mathcal{D} N\left(\xi_{1}(t)\right) \\
= & u_{M}(t)-C A^{-1}(A x(t)+B \mathcal{D} r(t)+F w(t)) \\
& -C A^{-1} B \mathcal{D} N\left(\xi_{1}(t)\right) \\
= & u_{M}(t)-C x(t)-C A^{-1} B \mathcal{D}\left(r(t)-N\left(\xi_{1}(t)\right)\right) \\
& -C A^{-1} F w(t) \\
= & -C A^{-1} F w(t)
\end{aligned}
$$

where the equalities $u_{M}=y=C x$ and $r=N\left(\xi_{1}\right)$ have been used. This implies that $z(t)=I_{1}$ for all $t \in\left[t_{0}, t_{1}\right], z(t)=I_{2}$ for all $t \in\left[t_{2},+\infty\right)$, and

$$
\begin{aligned}
I_{2}-I_{1} & =z\left(t_{2}\right)-z\left(t_{1}\right)=-C A^{-1} F \int_{t_{1}}^{t_{2}} w(t) d t \\
& =-C A^{-1} F \int_{t_{1}}^{t_{2}} \bar{w}(t) d t
\end{aligned}
$$

and hence (20) holds if and only if condition (51) is satisfied. If $A$ is singular, the same conclusion is readily obtained once $z$ is defined as

$z \doteq \bar{v} x+\bar{v} B N\left(\xi_{1}\right)$

and proceeding as above. In particular, it turns out that

$\mathcal{D} z(t)=-\bar{v} F w(t)$,

which leads to conclude that (20) holds if and only if condition (52) is satisfied. In the other two cases, i.e., $\Sigma_{\mathrm{ME}}=\Sigma_{\mathrm{ME}}^{(\mathrm{II})}$ and $\Sigma_{\mathrm{ME}}=\Sigma_{\mathrm{ME}}^{(\mathrm{III})}$, the proof follows along the same lines and is omitted.

Remark 7 From the proof of Proposition 4 it follows that if the control vector $F$ is chosen such that $C A^{-1} F=0$ (or $\bar{v} F=0$ in the singular case), then the system dynamics does not leave the initial manifold $\mathcal{M}_{1}$ but it remains confined onto $\mathcal{M}_{1}$ for whatever input $w$. Hence, $\operatorname{Ker}\left(C A^{-1}\right)$ (or $\left.\operatorname{Ker}(\bar{v})\right)$ contains all the control vectors $F$ which cannot be used to steer the system dynamics from one invariant manifold to another.

Proposition 4 shows that for $n>1$ there exists a onedimensional affine subspace of control vectors $F \in \mathbb{R}^{n}$ able to move the system dynamics from the initial manifold $\mathcal{M}_{1}$ to the final manifold $\mathcal{M}_{2}$ according to relation (20). Such a degree of freedom can be exploited to design $F$ in order to satisfy some additional control specifications. In particular, we are interested in investigating how $F$ can be designed to make its implementation easier. As shown in Sect. 5, each nonzero component of $F$ would require to insert an independent source into the two-terminal element $\mathbf{L}$. Hence, to minimize the number of sources needed to implement $F$, it can be chosen to have only one component different from zero, i.e.,

$F \in \operatorname{span}\left\{e_{i}\right\}$ 
where $e_{i} \in \mathbb{R}^{n}$ denotes the $i$-th versor of $\mathbb{R}^{n}$. Let $\left[C A^{-1}\right]_{i}, i \in J \subseteq\{1, \ldots, n\}$, denote the components of the vector $C A^{-1}$ which are different from zero. Then, condition (51) is satisfied if and only if $F$ is such that

$F \in\left\{\frac{1}{\left[C A^{-1}\right]_{i}} e_{i}, \quad i \in J\right\}$.

Similarly, if we denote by $[\bar{v}]_{i}, i \in J \subseteq\{1, \ldots, n\}$, the components of $\bar{v}$ different from zero, then condition (52) is satisfied if and only if $F$ is such that

$F \in\left\{\frac{1}{[\bar{v}]_{i}} e_{i}, \quad i \in J\right\}$.

Note that since both $C A^{-1}$ and $\bar{v}$ are nonzero vectors, the set $J$ is not empty and hence it is always possible to select $F$ enjoying the simplest structure in (60).

Another important issue in designing $F$ is how the piecewise-continuous control input $w(t)$ affects smoothness of the dynamics of the controlled system of Fig. $3 \mathrm{~b}$ with respect to the uncontrolled one of Fig. 3a. The following result pertaining to system $\Sigma_{\mathbf{L}}^{(c)}$ provides useful insights on this issue.

Proposition 5 Consider system $\Sigma_{\mathbf{L}}^{(c)}$ and let $w(t)$ be as in (19). Then, for any input signal $u(t)$ the output signal $y(t)$ and its time-derivatives $\mathcal{D} y(t), \mathcal{D}^{2} y(t), \ldots$, $\mathcal{D}^{n-1} y(t)$ do not explicitly depend on the control input $w(t)$ if and only if $F$ satisfies the following $n-1$ conditions:

$$
\begin{cases}C F & =0 \\ C A F & =0 \\ \cdots & =0 \\ C A^{n-2} F & =0 .\end{cases}
$$

Proof It can be verified that $\mathcal{D} y(t), \mathcal{D}^{2} y(t), \ldots$, $\mathcal{D}^{n-1} y(t)$ can be expressed in terms of $x(t), u(t)$ and $w(t)$ as follows:

$$
\left\{\begin{aligned}
y(t)= & C x(t)+D u(t) \\
\mathcal{D} y(t)= & C A x(t)+C B u(t)+D \mathcal{D} u(t)+C F w(t) \\
\mathcal{D}^{2} y(t)= & C A^{2} x(t)+C A B u(t)+C B \mathcal{D} u(t) \\
& +D \mathcal{D}^{2} u(t)+C A F w(t)+C F \mathcal{D} w(t) \\
\cdots & \cdots \\
\mathcal{D}^{n-1} y(t)= & C A^{n-1} x(t)+\sum_{k=0}^{n-2} C A^{n-2-k} B \mathcal{D}^{k} u(t) \\
& +D \mathcal{D}^{n-1} u(t) \\
& +\sum_{k=0}^{n-2} C A^{n-2-k} B^{k} w(t) .
\end{aligned}\right.
$$

Hence, while $y(t)$ does not explicitly depend on $w(t)$, its time-derivatives $\mathcal{D} y(t), \mathcal{D}^{2} y(t), \ldots, \mathcal{D}^{n-1} y(t)$ do not directly depend on the control input $w(t)$ if and only if conditions (63) hold.

Remark 8 It is important to note that if $F$ satisfies conditions (63), then smoothness properties of $y(t)$, $\mathcal{D} y(t), \mathcal{D}^{2} y(t), \ldots, \mathcal{D}^{n-1} y(t)$ do not depend on the piecewise-continuous control input $w(t)$. Said another way, the smoothness properties of $y(t), \mathcal{D} y(t), \mathcal{D}^{2} y(t)$, $\ldots, \mathcal{D}^{n-1} y(t)$ are the same pertaining to the uncontrolled system $\Sigma_{L}$. For instance, if $u(t)$ and its timederivatives $\mathcal{D} u(t), \mathcal{D}^{2} u(t), \ldots, \mathcal{D}^{n-1} u(t)$ are continuous, then the output of $\Sigma_{L}$ and its time-derivatives of order up to $n-1$ are continuous, while the output $\Sigma_{L}^{(c)}$ and its time-derivatives of order up to $n-1$ are continuous for any piecewise-continuous control input $w(t)$ if and only if $F$ satisfies conditions (63).

Remark 9 It is worth noting that conditions (63) are connected to a specific structural property of system $\Sigma_{L}^{(c)}$, i.e., strong observability [58]. Indeed, if conditions (63) hold then the state $x(t)$ is observable from the knowledge of the output $y(t)$ and the input $u(t)$ and their time-derivatives, even if $w(t)$ is unknown. This can be readily seen from equations (64) which, if conditions (63) hold, can be compactly rewritten as $\mathcal{O} x(t)=V(t)$, where $\mathcal{O}$ is the observability matrix (7) and $V(t)$ is a known vector since it depends on $y(t)$, $u(t)$ and their time-derivatives. Thus, Assumption 1 together with conditions (63) ensure that $x(t)$ can be observed even if $w(t)$ is unknown.

Proposition 5 and Remark 8 suggest that the choice of $F$ influences the smoothness of the output $y(t)$ of $\Sigma_{L}^{(c)}$ and hence smoothness of the input $u_{M}(t)$ of $\Sigma_{\mathrm{ME}}$. Indeed, it can be shown that if the ME nonlinear characteristic $N(\cdot)$ and its derivatives of order up to $k$ are continuous, then $F$ solving conditions (63) ensures that $y(t)$ and its time-derivatives of order up to $\min \{k, n-1\}$ are continuous. Conversely, if $F$ does not satisfy conditions (63), then Proposition 5 and Remark 8 make it clear that smoothness of $y(t)$ and its time-derivatives can be fulfilled only if $w(t)$ is allowed to be more smooth than piecewise-continuous. Hence, we are interested to investigate if there exists $F$ solving conditions (63) together with either (51) if $A$ is non-singular or (52) if $A$ is singular. The next result holds true.

Proposition 6 Let Assumption 1 hold. Then, 
- the unique control vector solving (63) and (51) is given by

$$
F=-A \mathcal{O}^{-1} e_{1} \doteq F_{n s},
$$

where $e_{1}=(1,0, \ldots, 0)^{\top} \in \mathbb{R}^{n}$ and $\mathcal{O}$ is the observability matrix (7);

- the unique control vector solving (63) and (52) is given by

$$
F=-\mathcal{Q}^{-1} e_{1} \doteq F_{S},
$$

where

$$
\mathcal{Q}=\left(\begin{array}{c}
\bar{v} \\
C \\
C A \\
\cdots \\
C A^{n-2}
\end{array}\right) \in \mathbb{R}^{n}
$$

Proof In the case of $A$ non-singular it is enough to observe that (63) can be rewritten as

$$
\begin{cases}C A A^{-1} F & =0 \\ C A^{2} A^{-1} F & =0 \\ \cdots & =0 \\ C A^{n-1} A^{-1} F & =0\end{cases}
$$

and hence (63) and (51) are satisfied if and only if

$\mathcal{O} A^{-1} F=-e_{1}$.

If $A$ is singular, it turns out that (63) and (52) are satisfied if and only if

$\mathcal{Q} F=-e_{1}$

and hence to complete the proof we need to show that $Q$ is non-singular. Proceeding by contradiction, suppose that det $Q=0$. This implies that there exist $\alpha_{k} \in \mathbb{R}$, $k=0, \ldots, n-2$, such that

$$
\bar{v}=\sum_{k=0}^{n-2} \alpha_{k} C A^{k} .
$$

Now, since $\bar{v} A=0$, it turns out that the following relationships

$0=\sum_{k=0}^{n-2} \alpha_{k} C A^{k+1}$

should hold. However, this would require that $C A, \ldots$, $C A^{n-1}$ are linearly dependent, which is a contradiction since $\mathcal{O}$ is non-singular.

\section{Control vector implementation in the two-terminal element $L$}

In this section, we investigate how the control vector $F$ can be implemented by suitably introducing voltage and current sources into the two-terminal element L (see Fig. 5). The results of Sects. 3 and 4 are valid for any state space representation of $\Sigma$ and $\Sigma_{L}^{(c)}$. In this section, we consider a specific state space representation, i.e., the one where the state vector is composed by the voltages $v_{C_{i}}$ of all the capacitors and the currents $i_{L_{i}}$ of all the inductors contained in $\mathbf{L}$. Specifically, if $\mathbf{L}$ has $n_{C}$ capacitors and $n_{L}$ inductors, then we assume that the state vector has the structure $x=\left(v_{C_{1}}, \ldots, v_{C_{n_{C}}}, i_{L_{1}}, \ldots, i_{L_{n_{L}}}\right) \in \mathbb{R}^{n}$ with $n=n_{C}+n_{L}$. The basic step to implement $F$ consists in introducing an independent current source $I_{i}^{(s)}$ in parallel to each capacitor with voltage $v_{C_{i}}$ and an independent voltage source $V_{i}^{(s)}$ in series to each inductor with current $i_{L_{i}}$. Said another way, each capacitor is replaced with its forced version, as shown in Fig. 6a, and each inductor is replaced with its forced version, as shown in Fig. 6b. It can be verified that, by applying the Kirchhoff's law to the loops and nodes of the two-terminal element, a state equation of the following structure can be derived

$\Sigma_{\mathbf{L}}^{(S)}:\left\{\begin{array}{l}\mathcal{D} x(t)=A x(t)+B u(t)+\Lambda^{-1} S \\ y(t)=C x(t)+D u(t),\end{array}\right.$

where $S \in \mathbb{R}^{n}$ is the vector of the voltage and current independent sources, i.e.,

$S=\left(I_{1}^{(s)}, \ldots, I_{n_{C}}^{(s)}, V_{1}^{(s)}, \ldots, V_{n_{L}}^{(s)}\right)^{\top}$, 
Fig. 6 a Each capacitor is replaced by its forced version (i.e., the parallel connection of the same capacitor with the current source $I^{(s)} ; \mathbf{b}$ Each inductor is replaced by its forced version (i.e., the series connection of the same inductor with the voltage source $V^{(s)}$ )

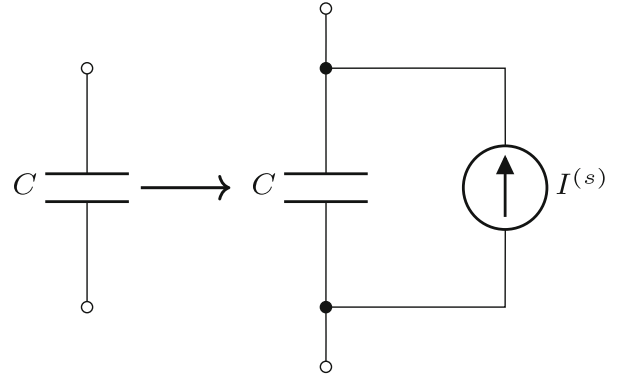

(a)

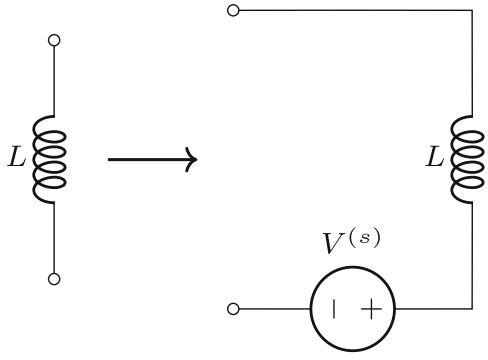

(b) and $\Lambda \in \mathbb{R}^{n \times n}$ is the following non-singular diagonal matrix

$\Lambda=\operatorname{diag}\left(C_{1}, \ldots, C_{n_{C}}, L_{1}, \ldots, L_{n_{L}}\right)$

with $C_{i}, i=1, \ldots, n_{C}$, and $L_{i}, i=1, \ldots, n_{L}$, being the capacitances of the capacitors and the inductances of the inductors, respectively. The last step for the controller implementation consists in making $\Sigma_{\mathbf{L}}^{(S)}$ equal to $\Sigma_{\mathbf{L}}^{(c)}$ via the relation

$S=\Lambda F w$

Hence, for any control vector $F$ satisfying either (51) or (52), relation (76) provides the corresponding implementation with a certain number of sources. It is important to underline that since $\Lambda$ is a diagonal matrix, only one source is needed when the control vector $F$ is selected as in either (61) or (62). This implies that the considered problem of steering the dynamics from an initial manifold to a final one within a given time interval can be always solved via the introduction of a unique source in the circuit.

\section{Application examples}

In this section, we consider some circuits of the class of Fig. 1 to illustrate the controller design and the implementation procedure discussed in Sects. 4 and 5, respectively. In the first two examples, the two-terminal element $\mathbf{L}$ is a second-order passive $R L C$ circuit and the mem-element $\mathbf{M E}$ is a memristor (specifically, $\mathbf{M} \mathbf{R}_{\varphi}$ in the first one and $\mathbf{M} \mathbf{R}_{q}$ in the second one). The third example considers the celebrated Chua's circuit with the nonlinear resistor (Chua's diode) replaced by

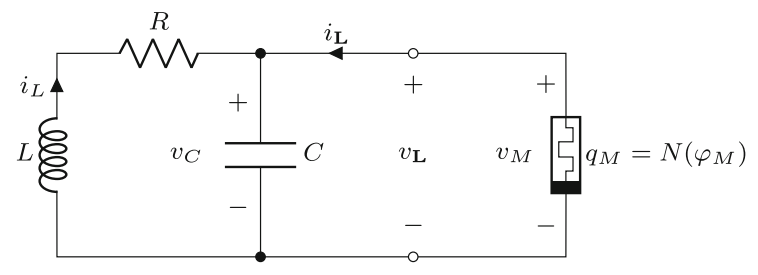

Fig. 7 Circuit of Example 1: a passive $R L C$ impedance connected to a flux-controlled memristor

a flux-controlled memristor $\mathbf{M R}_{\varphi}$. In the fourth example, the two-terminal element $\mathbf{L}$ is a second-order active circuit, whose impedance has a relative degree equal to 2 , while the mem-element is a flux-controlled memcapacitor $\mathbf{M C}_{\varphi}$. We also use this example to illustrate the procedure in the case when the matrix $A$ of $\Sigma_{\mathbf{L}}$ is singular. Finally, in the last example we consider the circuit introduced in [31] which has been shown to be physically controllable in the flux-charge domain [26]. The circuit consists of an active linear two-terminal element containing three capacitors and an operational amplifier interconnected with an ideal active voltage-controlled memristor.

Example 1 Consider the unforced version of the wellknown Murali-Lakshmanan-Chua oscillatory memristor circuit (see, e.g., [53]) depicted in Fig. 7. The twoterminal element $\mathbf{L}$ consists in the parallel connection of a capacitor $C$ with an inductor $L$ plus a resistor $R$, while the ME is a nonlinear flux-controlled memristor $\mathbf{M R}_{\varphi}$. It can be readily verified that $\mathbf{L}$ is described by the state equations

$\left\{\begin{array}{l}\mathcal{D} v_{C}(t)=\frac{1}{C} i_{L}(t)+\frac{1}{C} i_{\mathbf{L}}(t) \\ \mathcal{D} i_{L}(t)=-\frac{1}{L} v_{C}(t)-\frac{R}{L} i_{L}(t) \\ v_{\mathbf{L}}(t)=v_{C}(t),\end{array}\right.$ 
where $v_{C}$ is the capacitor voltage and $i_{L}$ is the inductor current. Hence, by assuming $x=\left(v_{C}, i_{L}\right)^{\top}, u=i_{\mathbf{L}}$ and $y=v_{\mathbf{L}}, \mathbf{L}$ admits the (current-voltage) representation $\Sigma_{\mathbf{L}}$ in (1) with the following matrices

$$
\begin{aligned}
A & =\left(\begin{array}{cc}
0 & \frac{1}{C} \\
-\frac{1}{L} & -\frac{R}{L}
\end{array}\right), & B & =\left(\begin{array}{c}
\frac{1}{C} \\
0
\end{array}\right), \\
C & =\left(\begin{array}{ll}
1 & 0
\end{array}\right), & D & =0 .
\end{aligned}
$$

Since the controllability and observability matrices in (6) and (7) boil down to

$$
\mathcal{R}=\left(\begin{array}{cc}
\frac{1}{C} & 0 \\
0 & -\frac{1}{L C}
\end{array}\right) \quad \mathcal{O}=\left(\begin{array}{ll}
1 & 0 \\
0 & \frac{1}{C}
\end{array}\right),
$$

it turns out that Assumption 1 holds. The real proper rational function $L(\mathcal{D})$ of the equivalent input-output description (2) of $\mathbf{L}$ is derived according to (3) as

$$
L(\mathcal{D})=\frac{\frac{1}{C} \mathcal{D}+\frac{R}{L C}}{\mathcal{D}^{2}+\frac{R}{L} \mathcal{D}+\frac{1}{L C}} .
$$

Note that the relative degree of $L(\mathcal{D})$ is equal to 1 , which implies that Assumption 2 holds. Hence, according to Tables 1 and 2, the circuit of Fig. 7 admits the feedback representation of Fig. 3a, where $\Sigma_{\mathbf{M E}}$ is given by $\Sigma_{\mathbf{M E}}^{(\mathrm{I})}$ in (8) with $\xi_{1}=\varphi_{M}, u_{M}=v_{M}, y_{M}=i_{M}$ and $N(\cdot)$ being the memristor flux-charge characteristic, i.e., $q_{M}=N\left(\varphi_{M}\right)$. Since $A$ is non-singular, according to Table 3 the invariant manifolds of the circuit of Fig. 7 are given by

$$
\begin{aligned}
\mathcal{M}_{I}= & \left\{\left(v_{C}, i_{L}\right)^{\top} \in \mathbb{R}^{2}, \varphi_{M} \in \mathbb{R}:\right. \\
& \left.\varphi_{M}+R C v_{C}+L i_{L}+R N\left(\varphi_{M}\right)=I\right\},
\end{aligned}
$$

where $I \in \mathbb{R}$ is the manifold index. As an example of the dynamics displayed on the manifolds, we refer to the case considered in [52] where the circuit parameters have the following normalized values:

$$
R=0.5 ; L=1 ; C=1
$$

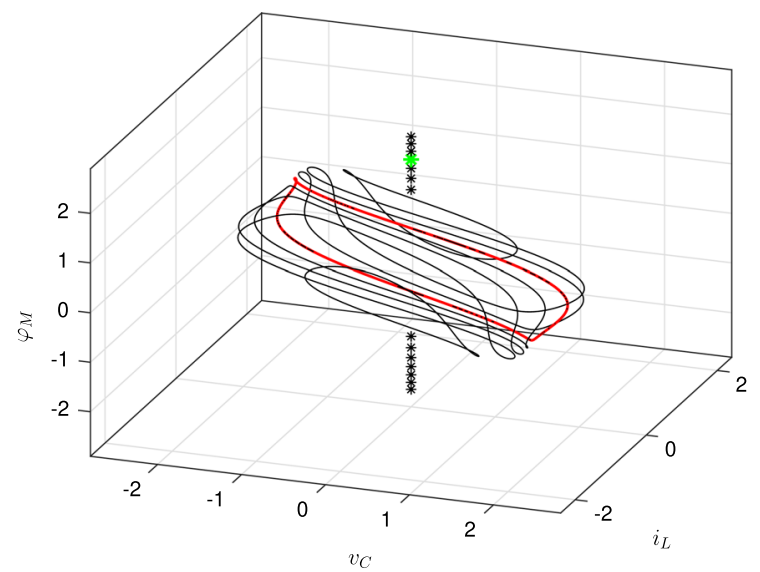

Fig. 8 Stable equilibrium points (marked with $\star$ ) and limit cycles (solid curves) for $I \in[-2.1,2.1]$. The limit cycle and the equilibrium point corresponding to $I=0.1$ and $I=1.5$ are drawn in red and green, respectively. (Color figure online)

and the flux-charge characteristic is given by

$q_{M}=N\left(\varphi_{M}\right)=-\varphi_{M}+0.1 \varphi_{M}^{3}$.

It turns out that each manifold $\mathcal{M}_{I}$ has a unique equilibrium point at $\left(v_{C}, i_{L}, \varphi_{M}\right)=\left(0,0, \bar{\varphi}_{M}\right)$ with $\bar{\varphi}_{M}$ such that $I=0.5 \bar{\varphi}_{M}\left(1+0.1 \bar{\varphi}_{M}^{2}\right)$ Moreover, the equilibrium point is the unique attractor of $\mathcal{M}_{I}$ if $|I|>0.755$, while for $|I|<0.755$ each $\mathcal{M}_{I}$ displays a stable limit cycle. Hence, the circuit has infinite stable limit cycles and equilibrium points and it undergoes to a Hopf bifurcation at $I= \pm 0.755$, which is referred to as a "bifurcation without parameters" since it is generated by varying the manifold index $I$, and hence by varying the initial conditions $\left(v_{C}\left(t_{0}\right), I_{L}\left(t_{0}\right), \varphi_{M}\left(t_{0}\right)\right)$, for fixed values of the circuit parameters. This dynamical scenario is reported in Fig. 8 where the specific attractors pertaining to $I=0.1$ (a limit cycle) and $I=1.5$ (an equilibrium point) are highlighted.

Consider now the controlled feedback system of Fig. $3 \mathrm{~b}$ where the matrices $A, B, C, D$ of $\Sigma_{\mathbf{L}}^{(c)}$ are as in (78) and the control input $w$ has the pulse shape in (19). According to Proposition 4, the control vectors $F=$ $\left(f_{1}, f_{2}\right)^{\top} \in \mathbb{R}^{2}$ able to move from the initial invariant manifold $\mathcal{M}_{1}$ pertaining to $I=I_{1}$, i.e., $\mathcal{M}_{1}=\mathcal{M}_{I_{1}}$, to the final invariant manifold $\mathcal{M}_{2}$ pertaining to $I=I_{2}$, i.e., $\mathcal{M}_{2}=\mathcal{M}_{I_{2}}$, must satisfy condition (51), i.e.,

$R C f_{1}+L f_{2}=1$. 


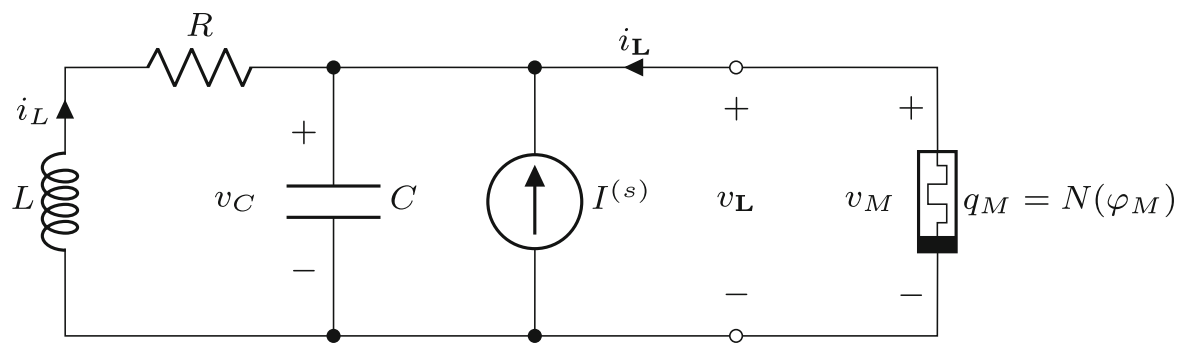

Fig. 9 Controlled circuit of Example 1 for $F=F_{1}$ : the capacitor is replaced by its forced version in Fig. 6a with $I^{(s)}=\frac{1}{R} w$.

Hence, the vectors $F$ solving the considered control problem for the circuit of Fig. 7 are given by a straight line in the $\left(f_{1}, f_{2}\right)$-plane. Among them, the vectors $F$ in (61) are given by the intersections of this straight line with the coordinate axes, i.e.,

$F \in\left\{\left(\begin{array}{c}\frac{1}{R C} \\ 0\end{array}\right),\left(\begin{array}{c}0 \\ \frac{1}{L}\end{array}\right)\right\} \doteq\left\{F_{1}, F_{2}\right\}$.

Moreover, the smooth control vector $F_{n s} \in \mathbb{R}^{2}$ defined in (65) of Proposition 6 satisfying conditions (63) of Proposition 5 is given by

$$
F_{n s}=\left(\begin{array}{cc}
0 & \frac{1}{C} \\
-\frac{1}{L} & -\frac{R}{L}
\end{array}\right)\left(\begin{array}{ll}
1 & 0 \\
0 & C
\end{array}\right)\left(\begin{array}{l}
1 \\
0
\end{array}\right)=\left(\begin{array}{l}
0 \\
\frac{1}{L}
\end{array}\right) \equiv F_{2} .
$$

To implement $F_{1}$ and $F_{2}=F_{n s}$ we proceed via the procedure described in Sect. 5. First, we note that the state vector $x=\left(v_{C}, i_{L}\right)^{\top}$ has already the right structure and that the source vector $S$ in (74) and the matrix $\Lambda$ in (75) boil down to

$$
S=\left(\begin{array}{c}
I^{(s)} \\
V^{(s)}
\end{array}\right)
$$

and

$$
\Lambda=\operatorname{diag}(C, L) \text {. }
$$

Hence, the control vectors $F$ in (85) can be implemented exploiting relation (76). For $F=F_{1}$ we get
In the case of $F=F_{2}=F_{n s}$ the controlled circuit is obtained by introducing, according to Fig. $6 \mathrm{~b}$, the voltage source $V^{(s)}=w$ in series to the inductor in the input-less circuit of Fig. 7

$V^{(s)}=0$ and

$I^{(s)}=\frac{1}{R} w$,

which implies that the controlled circuit contains a unique current source, as depicted in Fig. 9. For $F=$ $F_{2}=F_{n s}$ we get $I^{(s)}=0$ and

$V^{(s)}=w$,

and hence the input-less circuit is controlled by replacing the inductor with its forced version, according to Fig. 6b. In both cases $w(t)$ is as in (19) and satisfies (20).

To illustrate the behavior of the controlled circuit with $R, L, C$ and $N(\cdot)$ as in (82) and (83), we consider the problem of first steering the circuit dynamics from the initial manifold $\mathcal{M}_{1}=\mathcal{M}_{I_{1}}$ with $I_{1}=1.5$, which contains the stable equilibrium point marked in green in Fig. 8, to the final manifold $\mathcal{M}_{2}=\mathcal{M}_{I_{2}}$ with $I_{2}=0.1$, which contains the stable limit cycle drawn in red in Fig. 8, within a given time interval $\left[t_{1}, t_{2}\right]$, $t_{2}>t_{1}>t_{0}$ and then coming back to $\mathcal{M}_{1}$ within a time interval $\left[t_{3}, t_{4}\right], t_{4}>t_{3}>t_{2}$. According to (20), we need to employ a control input $w$ with a pulse of area equal to $I_{2}-I_{1}=-1.4$ in $\left[t_{1}, t_{2}\right]$ and to $I_{1}-I_{2}=1.4$ in $\left[t_{3}, t_{4}\right]$. The problem is first addressed by employing only the voltage source and then only the current source. Figure 10a and Fig. 10b report the pulse timing of the voltage and current source, respectively, together with the corresponding time behaviors of the manifold index $I$ and the controlled output $y=v_{C}$. Note that the output has a smoother behavior when the voltage source is used, as predicted by Proposition 6 .

Example 2 Consider the circuit of Fig. 11 where $\mathbf{L}$ consists in a series connection of a resistor $R$, an inductor 

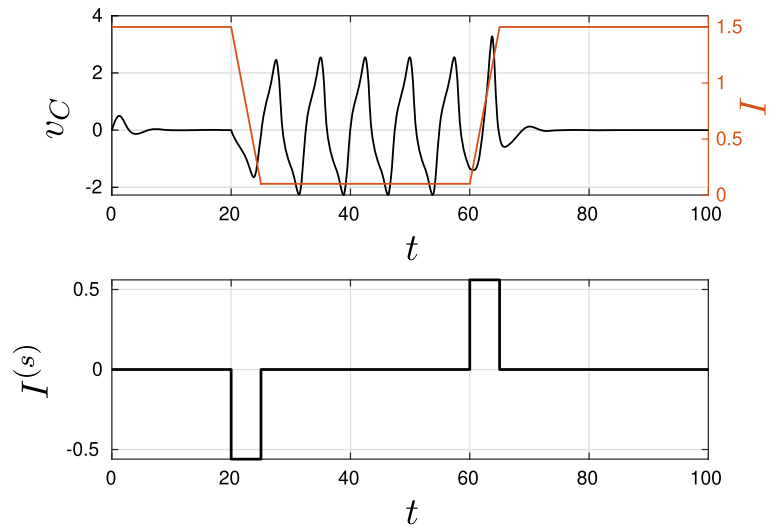

(a)

Fig. 10 a Circuit of Fig. 7 controlled as in Fig. 9. Lower plot: pulse timing of $I^{(s)}$; upper plot: time behaviors of the manifold index $I$ (red) and the controlled output $v_{C}$ (dark). b Circuit of Fig. 7 controlled by replacing the inductor with its forced version. Lower plot: pulse timing of $V^{(s)}$; upper plot: time behaviors of
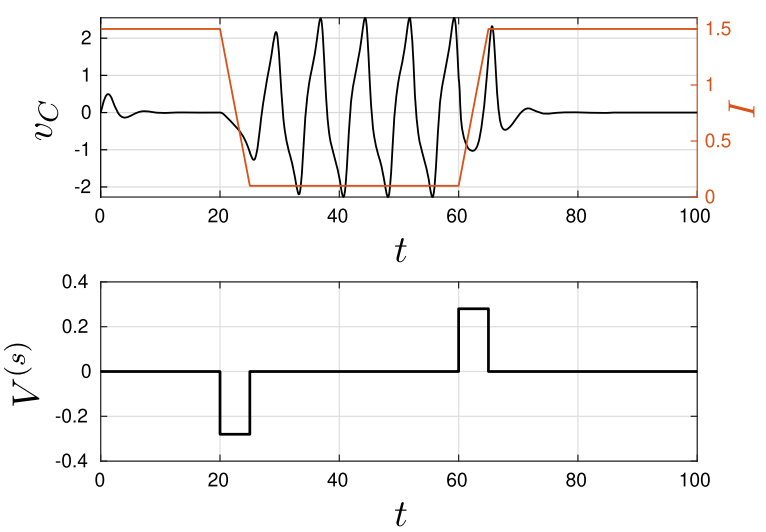

(b)

$I$ (red) and $v_{C}$ (dark). The width of all the rectangular pulses is equal to 5 arbitrary time units, while the area is equal to \pm 1.4 for $V^{(s)}$ and \pm 2.8 for $I^{(s)}$. The initial conditions are $v_{C}(0)=0.01$, $i_{L}(0)=0.62, \varphi_{M}(0)=1.45$
Fig. 11 Circuit of Example 2: a passive $R L C$ admittance connected to a charge-controlled memristor

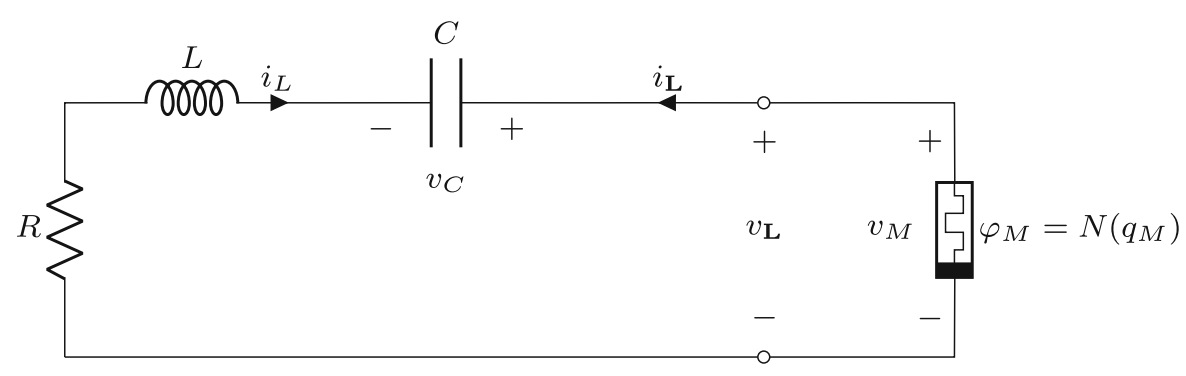

$L$, a capacitor $C$ and the $\mathbf{M E}$ is a nonlinear chargecontrolled memristor $\mathbf{M R}_{q}$. The two-terminal element is governed by the state equations

$$
\left\{\begin{array}{l}
\mathcal{D} v_{C}(t)=-\frac{1}{C} i_{L}(t) \\
\mathcal{D} i_{L}(t)=\frac{1}{L} v_{C}(t)-\frac{R}{L} i_{L}(t)-\frac{1}{L} v_{\mathbf{L}}(t) \\
i_{\mathbf{L}}(t)=-i_{L}(t)
\end{array}\right.
$$

where $v_{C}$ is the capacitor voltage and $i_{L}$ is the inductor current. Hence, by assuming $x=\left(v_{C}, i_{L}\right)^{\top}, u=-v_{\mathbf{L}}$ and $y=-i_{\mathbf{L}}$, it turns out that $\mathbf{L}$ admits the (voltagecurrent) representation $\Sigma_{\mathbf{L}}$ in (1) with

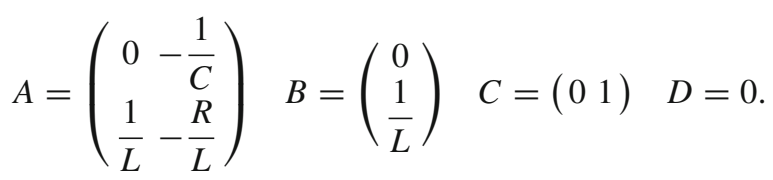

Since

$\mathcal{R}=\left(\begin{array}{cc}0 & -\frac{1}{L C} \\ \frac{1}{L} & -\frac{R}{L^{2}}\end{array}\right) \quad \mathcal{O}=\left(\begin{array}{cc}0 & 1 \\ \frac{1}{L} & -\frac{R}{L}\end{array}\right)$,

it turns out that Assumption 1 holds. Moreover, from (3) we get

$$
L(\mathcal{D})=\frac{\frac{1}{L} \mathcal{D}}{\mathcal{D}^{2}+\frac{R}{L} \mathcal{D}+\frac{1}{L C}},
$$

which implies that Assumption 2 holds. Hence, the circuit of Fig. 11 admits the feedback representation of Fig. 3a where $\Sigma_{\mathbf{M E}}$ is given by $\Sigma_{\mathbf{M E}}^{(\mathrm{I})}$ in (8) with $\xi_{1}=q_{M}, u_{M}=i_{M}, y_{M}=v_{M}$ and $N(\cdot)$ being the memristor charge-flux, i.e., $\varphi_{M}=N\left(q_{M}\right)$. Consequently, according to Table 3 , the circuit invariant 


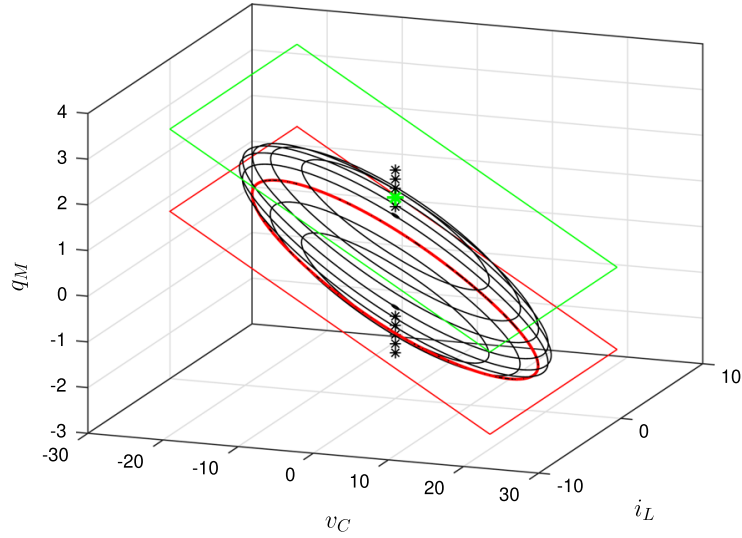

Fig. 12 Stable equilibrium points (marked with $\star$ ) and limit cycles (solid curves) for $I \in[-2,2]$. The limit cycle and the equilibrium point corresponding to $I=-0.4$ and $I=1.4$, and their associated manifolds, are drawn in red and green, respectively

manifolds are given by

$\mathcal{M}_{I}=\left\{\left(v_{C}, i_{L}\right)^{\top} \in \mathbb{R}^{2}, q_{M} \in \mathbb{R}: q_{M}+C v_{C}=I\right\}$.

Note that in this case the invariant manifolds are linear, as highlighted in Fig. (12).

As an example of the dynamics displayed on the manifolds, we refer to the specific circuit considered in [54] where the circuit parameters have the following normalized values

$R=0.4 ; L=1.5 ; C=0.1$

and the charge-flux characteristic is given by

$\varphi_{M}=N\left(q_{M}\right)=-0.7 q_{M}+0.3 q_{M}^{3}$.

It turns out that the scenario is quite similar to that of Example 1. Indeed, each manifold $\mathcal{M}_{I}$ has a unique equilibrium point at $\left(v_{C}, i_{L}, q_{M}\right)=(0,0, I)$, which is the unique attractor if $|I|>1$. If $|I|<1$, then the equilibrium point becomes unstable and the $\mathcal{M}_{I}$ displays a stable limit cycle, i.e., a Hopf bifurcation "without parameters" is likely to occur at $I= \pm 1$. Figure 12 depicts this dynamical scenario where the attractors pertaining to $I=-0.4$ (a limit cycle) and $I=1.4$ (an equilibrium point) and the relative invariant manifolds are highlighted.
In this case, condition (51) boils down to

$C f_{1}=1$,

and hence

$F_{1}=\left(\begin{array}{c}\frac{1}{C} \\ 0\end{array}\right)$

is the unique control vector $F$ which can be implemented with only one source. In fact, any control vector

$F_{2} \in \operatorname{span}\left\{\left(\begin{array}{l}0 \\ 1\end{array}\right)\right\}$

is such that $C A^{-1} F_{2}=0$ and hence, it cannot be used to move the dynamics from one invariant manifold to another (see Remark 7). From (65) we get the smooth control vector

$F_{n s}=-\left(\begin{array}{cc}0 & -\frac{1}{C} \\ \frac{1}{L} & -\frac{R}{L}\end{array}\right)\left(\begin{array}{ll}R & L \\ 1 & C\end{array}\right)\left(\begin{array}{l}1 \\ 0\end{array}\right)=\left(\begin{array}{c}1 \\ C \\ 0\end{array}\right) \equiv F_{1}$.

Taking into account that $x=\left(v_{C}, i_{L}\right)^{\top}$ and that $S=\left(I^{(s)}, V^{(s)}\right)^{\top}$ and $\Lambda=\operatorname{diag}(C, L)$, relation (76) reduces to $V^{(s)}=0$ and

$I^{(s)}=w$.

Hence, $F_{1}=F_{n s}$ is implemented by replacing the capacitor with its forced version. Finally, we note that any control vector $F_{2}$ satisfying (100) can be implemented by replacing the inductor with its forced version.

Let us now consider the same control problem of Example 1, i.e., steering the circuit dynamics from $\mathcal{M}_{1}=\mathcal{M}_{I_{1}}$ with $I_{1}=1.4$, which contains the stable equilibrium point drawn in green in Fig. 12, to $\mathcal{M}_{2}=\mathcal{M}_{I_{2}}$ with $I_{2}=-0.4$, which contains the stable limit cycle drawn in red in Fig. 12, and then coming back to $\mathcal{M}_{1}$.

This problem can be solved by using the current source (102) in parallel to the capacitor. As an example, we employ the control input $w$ with a rectangular pulse and a triangular pulse of area equal to -1.8 and 

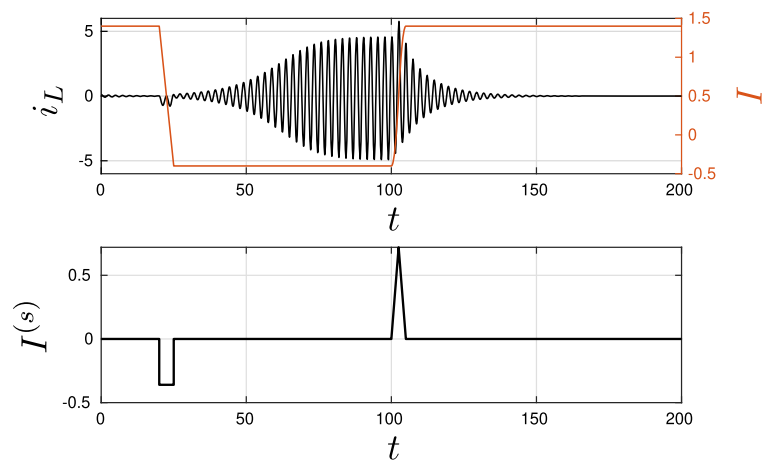

(a)

Fig. 13 a Circuit of Fig. 11 controlled by replacing the capacitor with its forced version: pulse timing of $I^{(s)}$ (lower plot). b Circuit of Fig. 11 is controlled by replacing the inductor with its forced version: pulse timing of $V^{(s)}$ (lower plot). The width of all the pulses is equal to 5 arbitrary time units, while the area is equal to
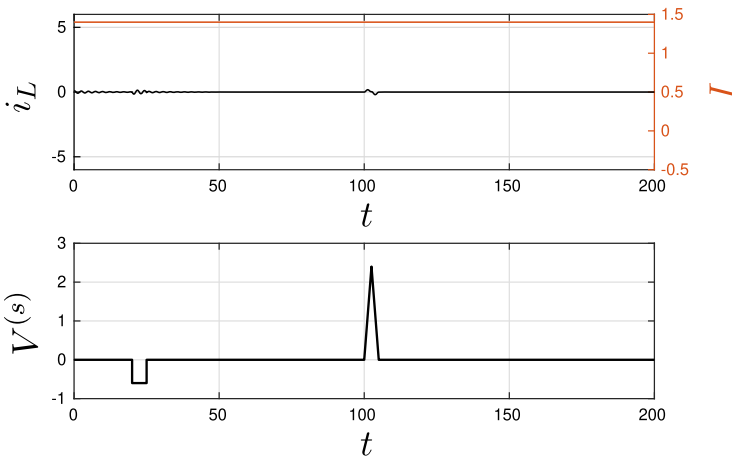

(b)

\pm 1.8 for $I^{(s)}$ and \pm 3 for $V^{(s)}$. Upper plots: corresponding time behaviors of the manifold index $I$ (red) and the controlled output $i_{L}$ (dark). The initial conditions are $v_{C}(0)=0.10, i_{L}(0)=0.10$, $q_{M}(0)=1.39$
Fig. 14 Circuit of Example 3: Chua's memristor circuit

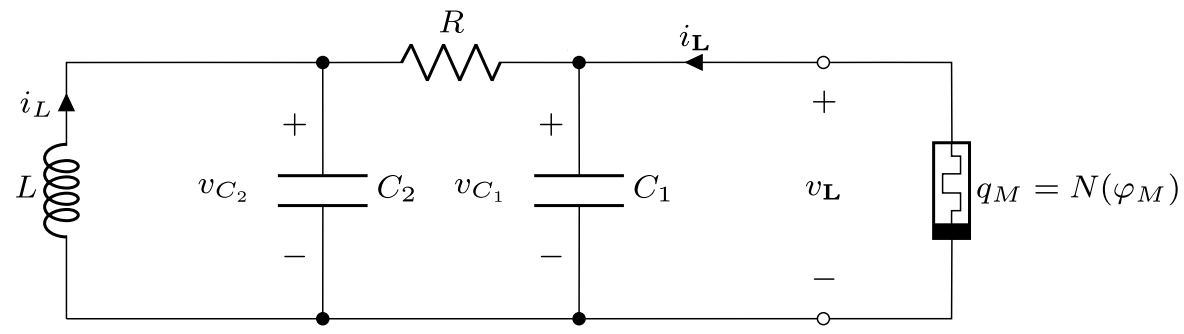

1.8 , respectively. The corresponding behaviors of the manifold index $I$ and the controlled output $y=i_{L}$ are depicted in Fig. 13a. Figure 13b reports the case when the input-less circuit is controlled via a voltage source in series to the inductor. Note that the index manifold $I$ does not change, i.e., as expected the dynamics remains onto $\mathcal{M}_{I_{1}}$.

Example 3 Consider the celebrated Chua's circuit reported in Fig. 14 with the nonlinear resistor (Chua's diode) replaced by a flux-controlled memristor $\mathbf{M R}_{\varphi}$. The two-terminal element $\mathbf{L}$, which contains two capacitors $C_{1}$ and $C_{2}$, one inductor $L$ and a resistor $R$, obeys the following equations:

$$
\left\{\begin{array}{l}
\mathcal{D} v_{C_{1}}(t)=-\frac{1}{R C_{1}} v_{C_{1}}+\frac{1}{R C_{1}} v_{C_{2}}+\frac{1}{C_{1}} i_{\mathbf{L}}(t) \\
\mathcal{D} v_{C_{2}}(t)=\frac{1}{R C_{2}} v_{C_{1}}-\frac{1}{R C_{2}} v_{C_{2}}+\frac{1}{C_{2}} i_{L}(t) \\
\mathcal{D} i_{L}(t)=-\frac{1}{L} v_{C_{2}}(t) \\
v_{\mathbf{L}}(t)=v_{C_{1}}(t)
\end{array}\right.
$$

where $v_{C_{1}}$ and $v_{C_{2}}$ are the capacitor voltages and $i_{L}$ is the inductor current. Hence, by assuming $x=$ $\left(v_{C_{1}}, v_{C_{2}}, i_{L}\right)^{\top}, u=i_{\mathbf{L}}$ and $y=v_{\mathbf{L}}$, the two-terminal element $\mathbf{L}$ admits the (current-voltage) representation $\Sigma_{\mathbf{L}}$ in (1) with

$A=\left(\begin{array}{ccc}-\frac{1}{R C_{1}} & \frac{1}{R C_{1}} & 0 \\ \frac{1}{R C_{2}} & -\frac{1}{R C_{2}} & \frac{1}{C_{2}} \\ 0 & -\frac{1}{L} & 0\end{array}\right), \quad B=\left(\begin{array}{c}\frac{1}{C_{1}} \\ 0 \\ 0\end{array}\right)$,

$C=\left(\begin{array}{lll}1 & 0 & 0\end{array}\right), \quad D=0$.

It can be readily verified that Assumption 1 holds and that the observability matrix becomes

$$
\mathcal{O}=\left(\begin{array}{ccc}
1 & 0 & 0 \\
-\frac{1}{R C_{1}} & \frac{1}{R C_{1}} & 0 \\
\frac{1}{R^{2} C_{1}}\left(\frac{1}{C_{1}}+\frac{1}{C_{2}}\right) & -\frac{1}{R^{2} C_{1}}\left(\frac{1}{C_{1}}+\frac{1}{C_{2}}\right) & \frac{1}{R C_{1} C_{2}}
\end{array}\right) .
$$


Also, Assumption 2 is satisfied since

$$
L(\mathcal{D})=\frac{\frac{1}{C_{1}}\left(\mathcal{D}^{2}+\frac{1}{R C_{2}} \mathcal{D}+\frac{1}{L C_{2}}\right)}{\mathcal{D}^{3}+\frac{C_{1}+C_{2}}{R C_{1} C_{2}} \mathcal{D}^{2}+\frac{1}{L C_{2}} \mathcal{D}+\frac{1}{R L C_{1} C_{2}}} .
$$

Note that Chua's memristor circuit admits the feedback representation of Fig. 3a, where $\Sigma_{\mathbf{M E}}$ is given by $\Sigma_{\mathbf{M E}}^{(\mathrm{I})}$ in (8) with $\xi_{1}=\varphi_{M}, u_{M}=i_{M}, y_{M}=v_{M}$ and $N(\cdot)$ being the memristor charge-flux characteristics, i.e., $q_{M}=N\left(\varphi_{M}\right)$. Hence, from Table 3 it follows that the circuit invariant manifolds are given by

$$
\begin{gathered}
\mathcal{M}_{I}=\left\{\left(v_{C_{1}}, v_{C_{2}}, i_{L}\right)^{\top} \in \mathbb{R}^{3}, \varphi_{M} \in \mathbb{R}:\right. \\
\left.\varphi_{M}+R C_{1} v_{C_{1}}+L i_{L}+R N\left(\varphi_{M}\right)=I\right\} .
\end{gathered}
$$

Let us consider the same circuit parameters and fluxcharge characteristic of [46]:

$R=1 ; L=1 / 15 ; C_{1}=1 / 10 ; C_{2}=1$,

$q_{M}=N\left(\varphi_{M}\right)=-\frac{8}{7} \varphi_{M}+\frac{4}{63} \varphi_{M}^{3}$.

The invariant manifolds $\mathcal{M}_{I}, I \in \mathbb{R}$, display quite a rich variety of attractors. For instance, the manifold $\mathcal{M}_{I}$ has a stable periodic solution for $I=-0.13$, while for $I_{2}=-0.03$ it has a more complex attractor which is generated via a classical period-doubling sequence by varying the index $I$ [46]. Both the attractors are depicted in Fig. 15.

According to Proposition 4, for the Chua's memristor circuit condition (51) becomes

$R C f_{1}+L f_{3}=1$.

Hence, the vectors $F$ in (61) which can be implemented via a unique source are

$\left.F \in\left\{\left(\begin{array}{c}\frac{1}{R C_{1}} \\ 0 \\ 0\end{array}\right),\left(\begin{array}{c}0 \\ 0 \\ \frac{1}{L}\end{array}\right)\right\} \doteq F_{1}, F_{2}\right\}$.

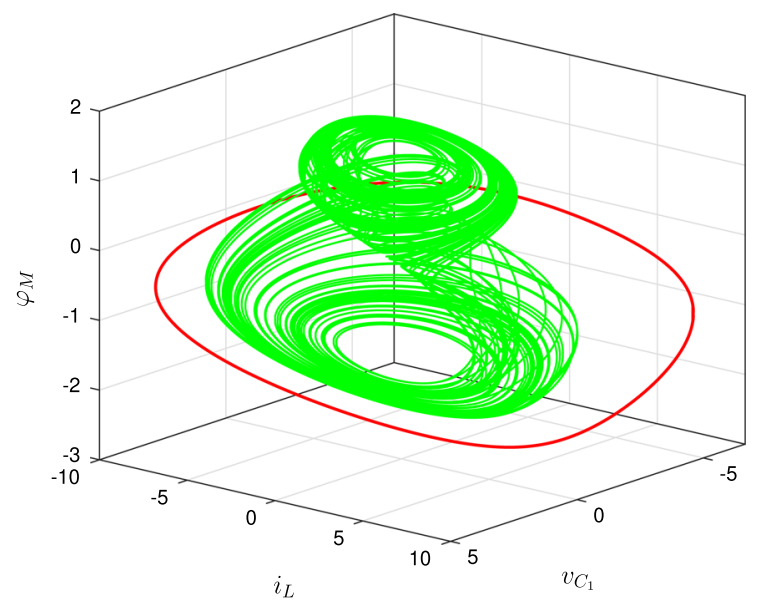

Fig. 15 Stable limit cycle (red) and complex attractor (green) in the $\left(v_{C_{1}}, i_{L}, \varphi_{M}\right)$-space for $I=-0.13$ and $I=-0.2$, respectively. (Color figure online)

Moreover, the smooth control vector $F_{n s} \in \mathbb{R}^{2}$ in (65) is given by

$F_{n s}=\left(\begin{array}{c}0 \\ 0 \\ \frac{1}{L}\end{array}\right) \equiv F_{2}$.

Since $x=\left(v_{C_{1}}, v_{C_{2}}, i_{L}\right)^{\top}, S=\left(I_{1}^{(s)}, I_{2}^{(s)}, V^{(s)}\right)^{\top}$ and $\Lambda=\operatorname{diag}\left(C_{1}, C_{2}, L\right)$, from (76) we get $I_{2}^{(s)}=V^{(s)}=$ 0 and

$I_{1}^{(s)}=\frac{1}{R} w$,

for $F=F_{1}$. Hence, $F_{1}$ is implemented via a unique current source. Conversely, for $F=F_{2}=F_{n s}$, we get $I_{2}^{(s)}=V^{(s)}=0$ and

$V^{(s)}=w$,

which implies that a unique voltage source is needed in this case. Finally, from Remark 7 it follows that the current source $I_{2}^{(s)}$ in parallel to the capacitor $C_{2}$ cannot be used to move the dynamics from one manifold to another.

Let us now consider the problem of steering the circuit dynamics from $\mathcal{M}_{1}=\mathcal{M}_{I_{1}}$ with $I_{1}=-0.13$, which contains the stable limit cycle of Fig. 15, to $\mathcal{M}_{2}=\mathcal{M}_{I_{2}}$ with $I=-0.03$, which contains the complex attractor of Fig. 15. The problem can be solved by using either 

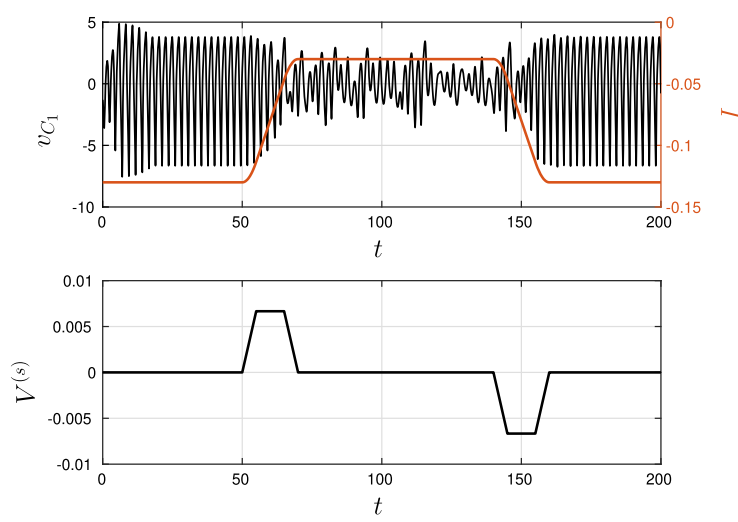

Fig. 16 Chua's memristor circuit controlled by replacing the inductor with its forced version. Lower plot: pulse timing $V^{(s)}$. The trapezoidal shaped pulses have an area of \pm 0.1 . Upper plot: time behaviors of the manifold index $I$ (red) and the controlled output $v_{C_{1}}$ (dark). The initial conditions are $v_{C_{1}}(0)=-1.3$, $v_{C_{2}}(0)=0, i_{L}(0)=0, \varphi_{M}(0)=0$

the current source (113) in parallel to the capacitor $C_{1}$ or the voltage source (114) in series to the inductor. As an example, we consider the case of the voltage source with trapezoidal shaped pulses. The pulse timing and the corresponding behaviors of the manifold index $I$ and the controlled output $v_{C_{1}}$ are depicted in Fig. 16.

Example 4 Consider the circuit of Fig. 17 where the two-terminal element contains two capacitors, five resistors and one operational amplifier, while the memelement $\mathbf{M E}$ is a flux-controlled memcapacitor $\mathbf{M C}_{\varphi}$. The structure of $\mathbf{L}$ has been chosen according to [59], in order to ensure that its impedance can be any second order filter with relative degree equal to 2 (see below). The two-terminal element obeys the following equations:

$$
\left\{\begin{array}{l}
\mathcal{D} v_{C_{1}}(t)=\frac{R_{2}-R_{1}}{R_{1} R_{2} C_{1}} v_{C_{1}}-\frac{1}{R_{2} C_{1}} v_{C_{2}} \\
\mathcal{D} v_{C_{2}}(t)=\frac{2 R_{2}-R_{1}}{R_{1} R_{2} C_{2}} v_{C_{1}}-\frac{R_{1}+R_{2}}{R_{1} R_{2} C_{2}} v_{C_{2}}-\frac{1}{C_{2}} i_{\mathbf{L}}(t) \\
v_{\mathbf{L}}(t)=2 v_{C_{1}}(t),
\end{array}\right.
$$

where $v_{C_{1}}$ and $v_{C_{2}}$ are the capacitor voltages. Hence, by assuming $x=\left(v_{C_{1}}, v_{C_{2}}\right)^{\top}, u=i_{\mathbf{L}}$ and $y=v_{\mathbf{L}}, \mathbf{L}$ admits the (current-voltage) representation $\Sigma_{\mathbf{L}}$ in (1) with

$$
A=\left(\begin{array}{cc}
\frac{R_{2}-R_{1}}{R_{1} R_{2} C_{1}} & -\frac{1}{R_{2} C_{1}} \\
\frac{2 R_{2}-R_{1}}{R_{1} R_{2} C_{2}} & -\frac{R_{1}+R_{2}}{R_{1} R_{2} C_{2}}
\end{array}\right), \quad B=\left(\begin{array}{c}
0 \\
-\frac{1}{C_{2}}
\end{array}\right),
$$

$C=(20)$,

$D=0$.

Assumption 1 and Assumption 2 hold since

$$
\mathcal{R}=\left(\begin{array}{cc}
0 & \frac{1}{2 R_{1} C_{1} C_{2}} \\
-\frac{1}{C_{2}} & \frac{R_{1}+R_{2}}{R_{1} R_{2} C_{2}^{2}}
\end{array}\right) \quad \mathcal{O}=\left(\begin{array}{cc}
2 & 0 \\
2 \frac{R_{2}-R_{1}}{R_{1} R_{2} C_{1}} & -2 \frac{1}{R_{2} C_{1}}
\end{array}\right)
$$

and

$$
L(\mathcal{D})=\frac{\frac{2}{R_{2} C_{1} C_{2}}}{\mathcal{D}^{2}+\frac{\left(R_{1}+R_{2}\right) C_{1}+\left(R_{1}-R_{2}\right) C_{2}}{R_{1} R_{2} C_{1} C_{2}} \mathcal{D}+\frac{2 R_{1}-R_{2}}{R_{1}^{2} R_{2} C_{1} C_{2}}} .
$$

As expected, the impedance of $\mathbf{L}$ has a constant at the numerator and a second-order polynomial at the denominator. Moreover, the coefficients of the numerator and denominator can be chosen arbitrarily by suitably selecting $C_{1}, C_{2}, R_{1}, R_{2}$.

It can be readily verified that the circuit of Fig. 17 admits the feedback representation of Fig. 3a with $u_{M}=v_{M}, y_{M}=i_{M}, u=i_{\mathbf{L}}$ and $y=v_{\mathbf{L}}$, while $\Sigma_{\mathbf{M E}}$ is given by $\Sigma_{\mathbf{M E}}^{(\mathrm{II})}$ in (9) with $\xi_{1}=\varphi_{M}, u_{M}=v_{M}$, $y_{M}=i_{M}$ and $N(\cdot)$ being the memcapacitor fluxcharge momentum characteristic, i.e., $\sigma_{M}=N\left(\varphi_{M}\right)$.

We observe that the matrix $A$ is not always nonsingular. In fact, it can be readily checked that its determinant vanishes when $R_{2}=2 R_{1}$. Hence, we proceed by separately considering the cases $A$ non-singular and $A$ singular.

Case I: $A$ non-singular, i.e., $R_{2} \neq 2 R_{1}$. From Table 3 it follows that the circuit invariant manifolds are given by

$$
\begin{aligned}
\mathcal{M}_{I}= & \left\{\left(v_{C_{1}}, v_{C_{2}}\right)^{\top} \in \mathbb{R}^{2}, \varphi_{M} \in \mathbb{R}:\right. \\
& \varphi_{M}+\frac{2\left(R_{1}+R_{2}\right) R_{1} C_{1}}{2 R_{1}-R_{2}} v_{C_{1}}-\frac{2 R_{1}^{2} C_{2}}{2 R_{1}-R_{2}} v_{C_{2}} \\
& \left.+\frac{4 R_{1}^{2}}{2 R_{1}-R_{2}} N^{\prime}\left(\varphi_{M}\right) v_{C_{1}}=I\right\} .
\end{aligned}
$$

Let us assume that the circuit parameters have the following normalized values:

$R_{1}=0.05 ; R_{2}=0.05 ; C_{1}=0.4 ; C_{2}=150$, 


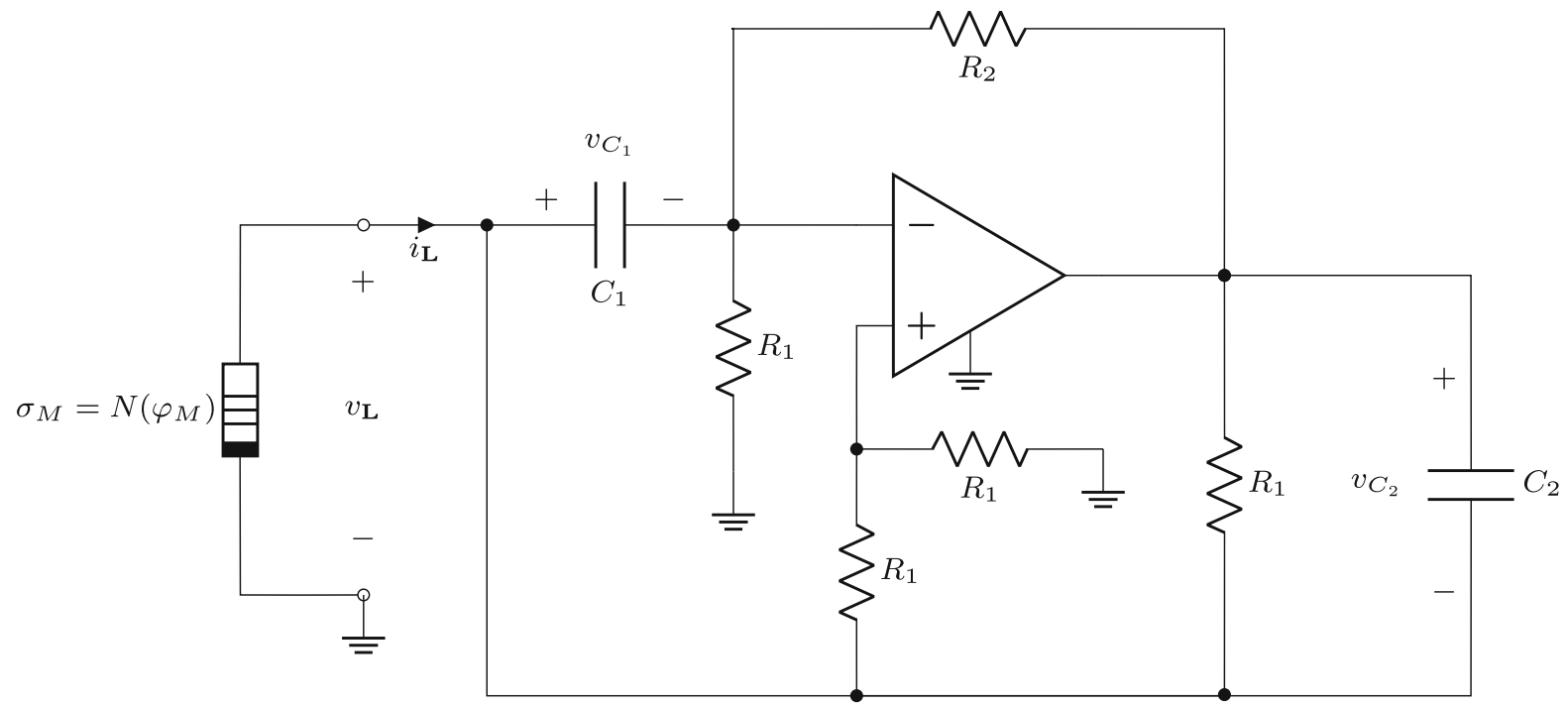

Fig. 17 Circuit of Example 4: $\mathbf{L}$ is an active two/terminal element; $\mathbf{M E}$ is a flux-controlled memcapacitor $\mathbf{M C} \mathbf{C}_{\varphi}$

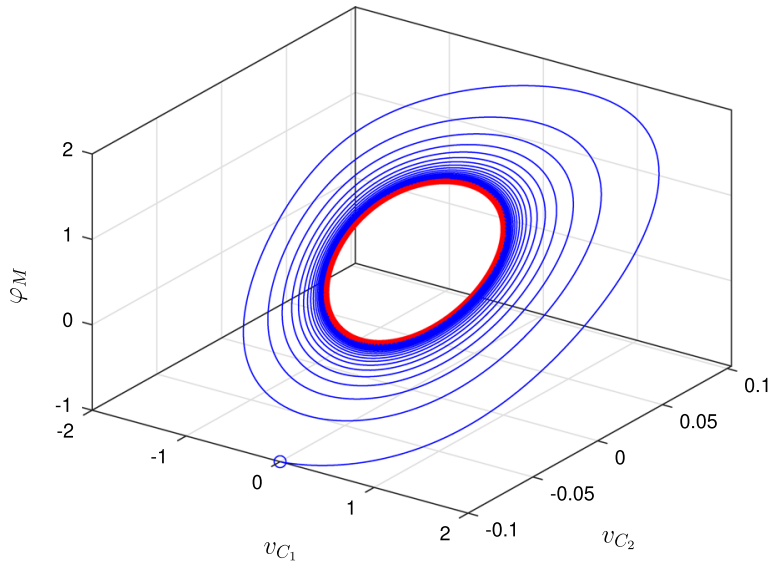

(a)

Fig. 18 a Stable limit cycle (red) on $\mathcal{M}_{I}$ for $I=0.5$. The initial conditions (marked with $\circ$ ) are $v_{C_{1}}(0)=0, v_{C_{2}}(0)=-0.1$, $\varphi_{M}(0)=-1$; b stable equilibrium point (green) on $\mathcal{M}_{I}$ for

and the nonlinear characteristic of the flux-controlled memcapacitor is given by:

$\sigma_{M}=N\left(\varphi_{M}\right)=-0.7 \varphi_{M}+0.1 \varphi_{M}^{3}$.

The corresponding invariant manifolds (119) exhibit either stable equilibrium points or stable limit cycles depending on the value of the index $I$. For instance, a stable periodic solution and a stable equilibrium point

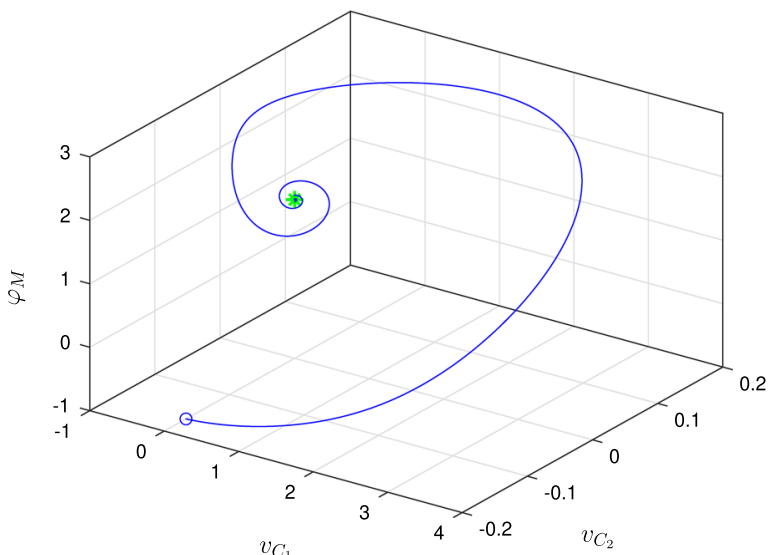

(b)

$I=1.5$. The initial conditions (marked with $\circ$ ) are $v_{C_{1}}(0)=0$, $v_{C_{2}}(0)=-0.17, \varphi_{M}(0)=-1$

are displayed for $I_{1}=0.5$ and $I_{2}=1.5$, respectively, as depicted in Fig. 18.

Now, condition (52) of Proposition 4 boils down to

$\frac{2\left(R_{1}+R_{2}\right) R_{1} C_{1}}{2 R_{1}-R_{2}} f_{1}-\frac{2 R_{1}^{2} C_{2}}{2 R_{1}-R_{2}} f_{2}=1$.

Hence, the control vectors $F$ able to steer the circuit dynamics from one invariant manifold to another via a 
unique source are

$F \in\left\{\left(\begin{array}{c}\frac{2 R_{1}-R_{2}}{2\left(R_{1}+R_{2}\right) R_{1} C_{1}} \\ 0\end{array}\right), \quad\left(\begin{array}{c}0 \\ -\frac{2 R_{1}-R_{2}}{2 R_{1}^{2} C_{2}}\end{array}\right)\right\} \doteq\left\{F_{1}, F_{2}\right\}$.

Moreover, from (65) we get the smooth control vector as

$F_{n s}=\left(\begin{array}{c}0 \\ -\frac{2 R_{1}-R_{2}}{2 R_{1}^{2} C_{2}}\end{array}\right) \equiv F_{2}$.

Since $x=\left(v_{C_{1}}, v_{C_{2}}\right)^{\top}$, while $S=\left(I_{1}^{(s)}, I_{2}^{(s)}\right)^{\top}$ and $\Lambda=\operatorname{diag}\left(C_{1}, C_{2}\right)$, for $F=F_{1}$ we get $I_{2}^{(s)}=0$ and

$I_{1}^{(s)}=\frac{2 R_{1}-R_{2}}{2\left(R_{1}+R_{2}\right) R_{1}} w$,

while for $F=F_{2}=F_{n s}$ we have $I_{1}^{(s)}=0$ and

$I_{2}^{(s)}=-\frac{2 R_{1}-R_{2}}{2 R_{1}^{2}} w$.

Hence, both $F_{1}$ and $F_{2}=F_{n s}$ are implement via a unique current source. Consider the problem of steering the circuit dynamics from $\mathcal{M}_{1}=\mathcal{M}_{I_{1}}$ with $I_{1}=1.5$, which contains the stable equilibrium point of Fig. 18, to $\mathcal{M}_{2}=\mathcal{M}_{I_{2}}$ with $I_{2}=0.5$, which contains the stable limit cycle of Fig. 18. The problem can be solved by replacing any capacitor of the input-less circuit in Fig. 17 with its forced version. As an example, we consider the case when the current source (126) is employed with $w$ having rectangular shaped pulses. Figure 19 reports the behaviors of the manifold index $I$ and the controlled output of (115).

Case II: $A$ singular, i.e., $R_{2}=2 R_{1}$. In this case the matrix $A$ boils down to

$A=\left(\frac{\frac{1}{2 R_{1} C_{1}}-\frac{1}{2 R_{1} C_{1}}}{\frac{3}{2 R_{1} C_{2}}-\frac{3}{2 R_{1} C_{2}}}\right)$

According to Proposition 2, to obtain the invariant manifolds we first need to compute $\bar{v}$ such that $\bar{v} A=0$. We get

$\bar{v}=\left(\begin{array}{ll}-3 C_{1} & C_{2}\end{array}\right)$,
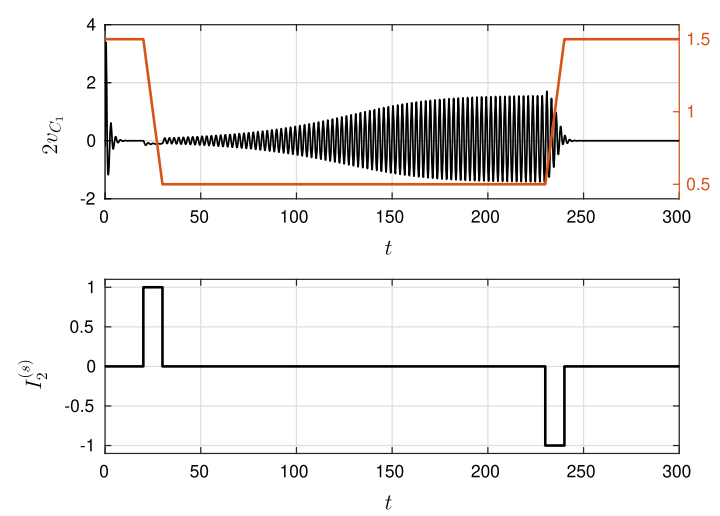

Fig. 19 Circuit of Fig. 17 controlled by replacing the capacitor $C_{2}$ with its forced version. Lower plot: pulse timing $I_{2}^{(s)}$. The rectangular shaped pulses have a width equal to 10 arbitrary time units and an amplitude of \pm 1 . Upper plot: time behaviors of the manifold index $I$ (red) and the controlled output of (115) (dark). The initial conditions are $v_{C_{1}}(0)=-0.1, v_{C_{2}}(0)=-0.09$, $\varphi_{M}(0)=0.1$

which leads to the following expression of the invariant manifolds

$$
\begin{aligned}
\mathcal{M}_{I}= & \left\{\left(v_{C_{1}}, v_{C_{2}}\right)^{\top} \in \mathbb{R}^{2}, \varphi_{M} \in \mathbb{R}:\right. \\
& \left.-3 C_{1} v_{C_{1}}+C_{2} v_{C_{2}}-2 N^{\prime}\left(\varphi_{M}\right) v_{C_{1}}=I\right\} .
\end{aligned}
$$

According to Proposition 4, the control vectors $F=$ $\left(f_{1}, f_{2}\right)^{\top} \in \mathbb{R}^{2}$ able to steer the circuits dynamics from one manifold to another, are such that

$3 C_{1} f_{1}-C_{2} f_{2}=1$.

Hence, the vectors $F$ in (62) requiring a unique source are

$F \in\left\{\left(\begin{array}{c}\frac{1}{3 R C_{1}} \\ 0\end{array}\right),\left(\begin{array}{c}0 \\ -\frac{1}{C_{2}}\end{array}\right)\right\} \doteq\left\{F_{1}, F_{2}\right\}$,

while from (66) we obtain the smooth control vector

$$
\begin{aligned}
F_{s}= & -\mathcal{Q}^{-1} e_{1}=\left(\begin{array}{cc}
-3 C_{1} & C_{2} \\
-2 & 0
\end{array}\right)^{-1}\left(\begin{array}{l}
1 \\
0
\end{array}\right) \\
& =\left(\begin{array}{c}
0 \\
-\frac{1}{C_{2}}
\end{array}\right) \equiv F_{2} .
\end{aligned}
$$


Fig. 20 Circuit of Example 5: $\mathbf{L}$ is an active two-terminal element; $\mathbf{M E}$ is an ideal active voltage-controlled (or flux-controlled) memristor with state variable $V_{0}$

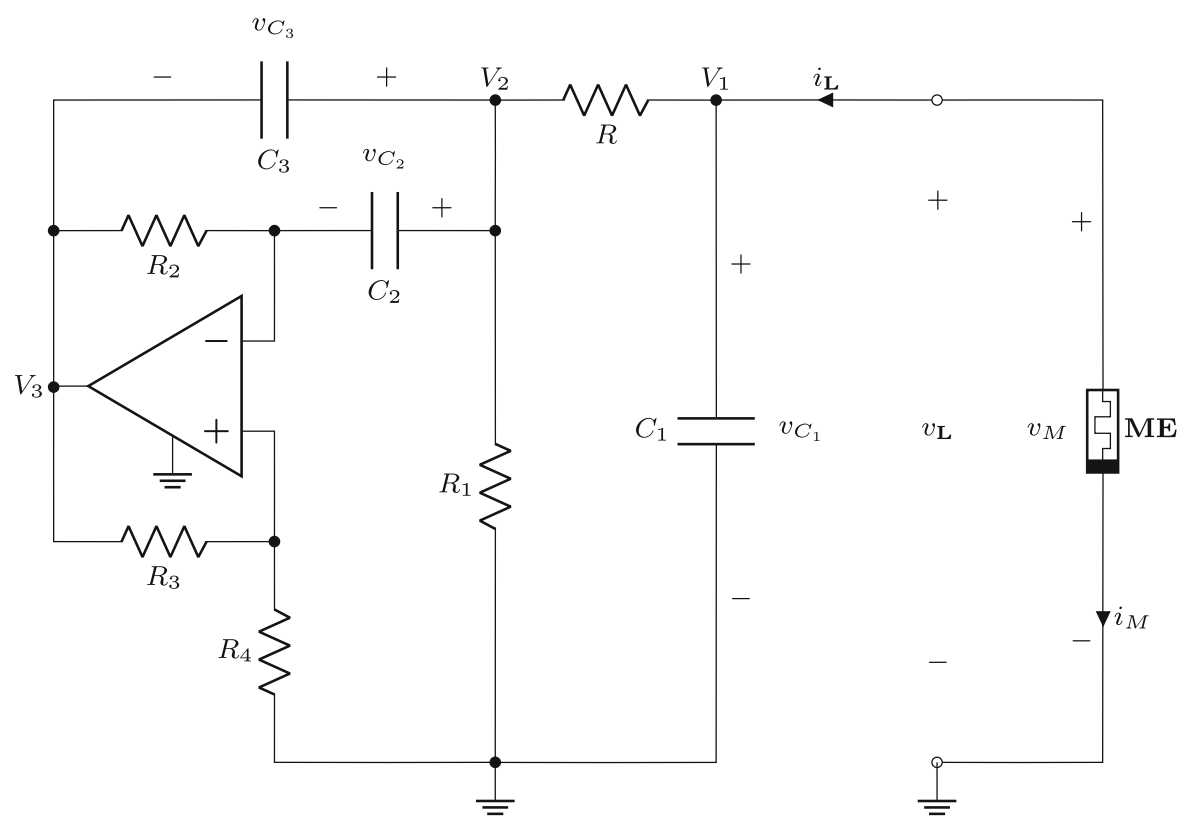

To implement both $F_{1}$ and $F_{2}=F_{s}$, we proceed as in Case I. For $F=F_{1}$ we get

$$
\left(\begin{array}{l}
I_{1}^{(s)} \\
I_{2}^{(s)}
\end{array}\right)=\left(\begin{array}{c}
1 \\
3 \\
0
\end{array}\right) w,
$$

while for $F=F_{2}=F_{s}$

$$
\left(\begin{array}{l}
I_{1}^{(s)} \\
I_{2}^{(s)}
\end{array}\right)=\left(\begin{array}{c}
0 \\
-1
\end{array}\right) w .
$$

Example 5 Consider the circuit of Fig. 20 introduced in [31] which is given by the interconnection via an $R C$ element of a single amplifier biquad-based active band-pass filter and an ideal active voltage-controlled (or flux-controlled) memristor. In [26], multistability is analyzed in the flux-charge domain by showing that it can be controlled by tuning the initial conditions of the voltages $V_{0}, V_{1}, V_{2}$, and $V_{3}$. The equations governing the circuit dynamics are [26]
$\left\{\begin{array}{l}\mathcal{D} V_{0}(t)=-\frac{1}{R_{a} C_{0}} V_{1} \\ \mathcal{D} V_{1}(t)=-\frac{1}{R C_{1}} V_{1}+\frac{1}{R C_{1}} V_{2}-\frac{1}{C_{1}} i_{M}(t) \\ \mathcal{D} V_{2}(t)=-\frac{k}{R C} V_{1}+\frac{k}{R^{\prime} C} V_{2}-\frac{2 k+1}{(k+1) R_{2} C} V_{3} \\ \mathcal{D} V_{3}(t)=-\frac{k+1}{R C} V_{1}+\frac{k+1}{R C} V_{2}-\frac{2}{R_{2} C} V_{3} \\ i_{M} \quad=W\left(V_{0}\right) V_{1}=\frac{1}{R_{b}}\left(-1+g V_{0}^{2}\right) V_{1}\end{array}\right.$

where $k \doteq R_{4} / R_{3}, R^{\prime} \doteq R R_{1} /\left(R+R_{1}\right), g$ is the total gain of the multipliers $M_{a}$ and $M_{b}$ and $C_{2}=C_{3}=C$.

Here, we want to show how the procedure developed in Sects. 4 and 5 can be applied to this circuit. To proceed, the matrices of the (current-voltage) state space representation $\Sigma_{\mathbf{L}}$ of $\mathbf{L}$ in (1) with $x=$ $\left(v_{C_{1}}, v_{C_{2}}, v_{C_{3}}\right)^{\top}, u=i_{\mathbf{L}}$ and $y=v_{\mathbf{L}}$ are first derived

$$
\begin{array}{ll}
A=\left(\begin{array}{ccc}
-\frac{1}{R C_{1}} & \frac{1+k}{R C_{1}} & -\frac{k}{R C_{1}} \\
0 & -\frac{1}{R_{2} C_{2}} & \frac{1}{R_{2} C_{2}} \\
\frac{f}{R^{\prime} C_{3}} & \frac{h-1-k}{R^{\prime} C_{3}} & \frac{k-h}{R^{\prime} C_{3}}
\end{array}\right), & B=\left(\begin{array}{c}
\frac{1}{C_{1}} \\
0 \\
0
\end{array}\right), \\
C=\left(\begin{array}{lll}
1 & 0 & 0
\end{array}\right), & D=0 .
\end{array}
$$




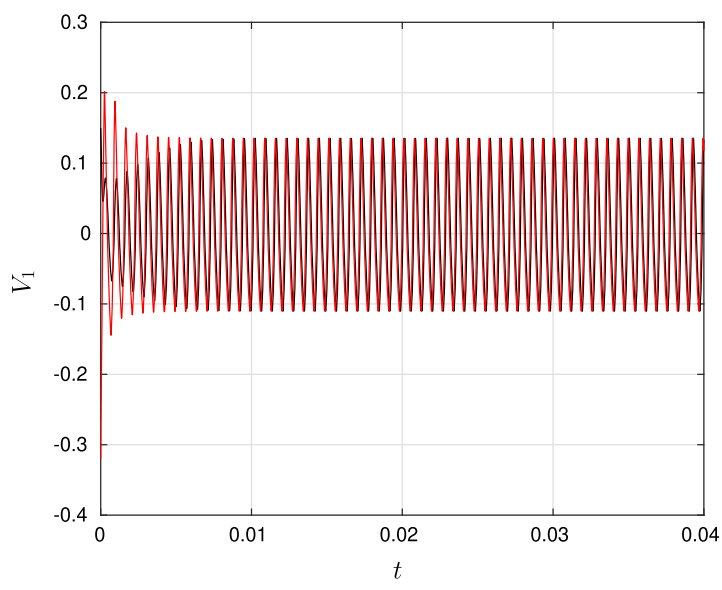

(a)

Fig. 21 a) Time behaviors of $V_{1}$; b) time behaviors of the manifold index $I$. The dark curves are generated by the initial conditions $V_{0}(0)=-1, V_{1}(0)=0.15, V_{2}(0)=0, V_{3}(0)=0$, while the red ones by the initial conditions $V_{0}(0)=-1.5$,

where $f=R^{\prime} / R$ and $h=R^{\prime} / R_{2}$. It can be readily verified that $A$ is non-singular and that Assumptions 1 and 2 hold. Also, we note that $v_{C_{1}}, v_{C_{2}}, v_{C_{3}}$ are related to $V_{1}, V_{2}, V_{3}$ as follows:

$$
\left\{\begin{array}{l}
v_{C_{1}}=V_{1} \\
v_{C_{2}}=V_{2}-\frac{k}{k+1} V_{3} \\
v_{C_{3}}=V_{2}-V_{3}
\end{array} .\right.
$$

Then, we observe that the ideal active voltagecontrolled memristor in Fig. 20 can be represented via $\Sigma_{\mathbf{M E}}^{(\mathrm{I})}$ in (8). Indeed, it is enough to define the flux $\varphi_{M}$ as follows

$\varphi_{M} \doteq-R_{a} C_{0} V_{0}$

and to assume the following flux-charge characteristics

$q_{M}=N\left(\varphi_{M}\right)=-\frac{R_{a} C_{0}}{R_{b}}\left(\frac{\varphi_{M}}{R_{a} C_{0}}-\frac{g}{3}\left(\frac{\varphi_{M}}{R_{a} C_{0}}\right)^{3}\right)$.

It turns that the circuit of Fig. 20 admits the feedback representation of Fig. 3a with $\Sigma_{\mathbf{L}}$ in (1) described by the matrices in (136) and $u=i_{\mathbf{L}}, y=v_{\mathbf{L}}$, and $\Sigma_{\mathbf{M E}}$ given by $\Sigma_{\mathbf{M E}}^{(\mathrm{I})}$ in (8) with $u_{M}=v_{M}, y_{M}=i_{M}, \xi$
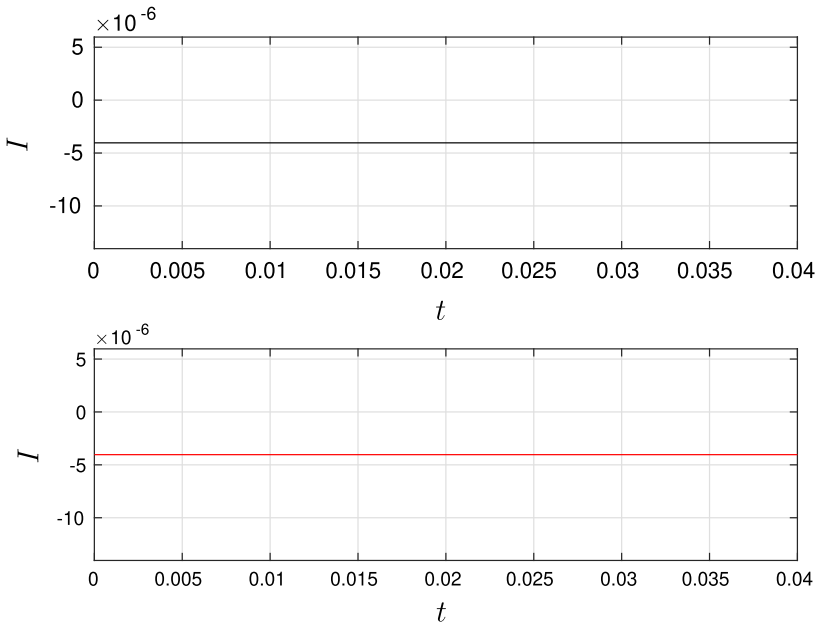

(b)

$V_{1}(0)=-0.325, V_{2}(0)=-0.02, V_{3}(0)=-0.3062$. Both the initial conditions belong to the invariant manifold in (141) with $I=4.0381 \cdot 10^{-6}$

equal to $\varphi_{M}$ in (138) and $N(\cdot)$ as in (139). Hence, from Table 3 it follows that the circuit invariant manifolds $\mathcal{M}_{I}$ are described by the following equation

$$
\begin{gathered}
\varphi_{M}-\left[C A^{-1}\right]_{1} v_{C_{1}}-\left[C A^{-1}\right]_{2} v_{C_{2}}-\left[C A^{-1}\right]_{3} v_{C_{3}} \\
+C A^{-1} B \frac{R_{a} C_{0}}{R_{b}}\left(\frac{\varphi_{M}}{R_{a} C_{0}}-\frac{g}{3}\left(\frac{\varphi_{M}}{R_{a} C_{0}}\right)^{3}\right)=I,
\end{gathered}
$$

where $\left[C A^{-1}\right]_{i}, i=1,2,3$, are the components of $C A^{-1}$. By exploiting the equations (137) and (138), the invariant manifolds can be equivalently expressed in terms of the original voltages $V_{0}, V_{1}, V_{2}, V_{3}$. Specifically, if we assume for the circuit parameters the values used in [26,31], i.e., $R_{a}=10 \mathrm{k} \Omega, R_{b}=1.4 \mathrm{k} \Omega$, $R=1.5 \mathrm{k} \Omega, R_{1}=100 \Omega, R_{2}=10 \mathrm{k} \Omega, R_{3}=1 \mathrm{k} \Omega$, $R_{4}=50 \Omega C_{0}=C_{1}=5 \mathrm{nF}, C 2=C 3=100 \mathrm{nF}$, $g=0.1$, we get

$$
\begin{aligned}
\mathcal{M}_{I}= & \left\{\left(V_{0}, V_{1}, V_{2}, V_{3}\right)^{\top} \in \mathbb{R}^{4}:\right. \\
& \left.\alpha_{0} V_{0}+\alpha_{1} V_{1}+\alpha_{2} V_{2}+\alpha_{3} V_{3}+\beta V_{0}^{3}=I\right\},
\end{aligned}
$$

with $\alpha_{0}=7.1429 \times 10^{-6}, \alpha_{1}=8 \times 10^{-6}, \alpha_{2}=2 \times$ $10^{-5}, \alpha_{3}=-1.0476 \times 10^{-5} \times, \beta=-1.9048 \times 10^{-6}$. 


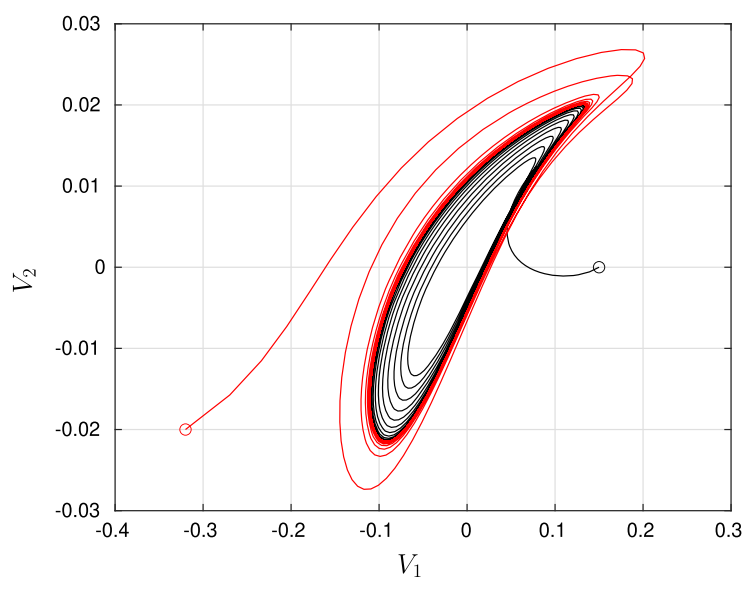

Fig. 22 Trajectories on the $\left(V_{1}, V_{2}\right)$ plane. The dark curves are generated by the initial conditions $V_{0}(0)=-1, V_{1}(0)=0.15$, $V_{2}(0)=0, V_{3}(0)=0$, while the red ones by the initial conditions $V_{0}(0)=-1.5, V_{1}(0)=-0.325, V_{2}(0)=-0.02$, $V_{3}(0)=-0.3062$. Both the initial conditions belong to the invariant manifold in (141) with $I=4.0381 \cdot 10^{-6}$

In [26] a thorough dynamical analysis has been performed in the flux-charge domain making it clear the strong multistable nature of the circuit, i.e., the coexistence of infinitely many different attractors.

Clearly, each attractor belongs to one of the infinite invariant manifolds in (141). As an example, if we consider the initial conditions $V_{0}(0)=-1, V_{1}(0)=0.15$, $V_{2}(0)=0, V_{3}(0)=0$, which are those of Fig. 3.b in [26] with $\delta=-1$, we obtain the behaviors of the voltage $V_{1}$ and the index $I$ reported (dark curves) in Fig. 21a and $b$, respectively. As expected, the index remains constant at the value $I=4.0381 \cdot 10^{-6}$. The corresponding trajectory in the $\left(V_{1}, V_{2}\right)$-plane converges toward a periodic solution (see Fig. 22). The three figures also report the behaviors (in red) generated by starting from different initial conditions which still belong to the same invariant manifold. Note that the index remains constant to the same value of $I=4.0381 \cdot 10^{-6}$, as expected.

Finally, since condition (51) boils down to

$$
0.8 f_{1}+f_{2}+f_{3}=10^{5}
$$

it follows that it is possible to steer the dynamics from an initial manifold to a final one within a given time interval by substituting any capacitor with its forced version. For instance, exploiting the implementation procedure of Sect. 5, it turns out that the following pulse-programmed current source

$I_{1}^{(s)}=6.25 \times 10^{-4} w$,

with the pulse $w$ chosen such that (20) holds, should be introduced in parallel to the capacitor $C_{1}$.

\section{Conclusions}

In this paper, the multistability control problem is considered for a class of circuits composed of the interconnection of a linear two-terminal (one-port) element and an ideal mem-element. Specifically, the problem of designing pulse programmed independent sources in order to steer the circuit dynamics onto one of the infinitely many invariant manifolds constituting the circuit state space is addressed. The linear two-terminal element can be either passive or active, while the memelement can be a flux- or charge-controlled memristor, a flux- or $\sigma$-controlled capacitor, a $\rho$ - or chargecontrolled inductor.

It is first shown that each circuit of the considered class admits a suitable feedback system representation, which permits to obtain an analytic expression of the infinitely many invariant manifolds constituting the circuit state space. Notably, the invariant manifolds are directly expressed in terms of the state variables of the two-terminal element and the mem-element, i.e., without resorting to the flux-charge approach. Moreover, the invariant manifolds are parameterized via a scalar parameter, which is referred to as the manifold index and is related to the circuit initial conditions. Then, the pulse shaped control inputs capable of steering the circuit dynamics from one initial manifold to a final one, within a given finite time interval and without modifying the attractors on the manifolds, are characterized. These pulse control inputs, which are programmed to have in the time interval an area equal to the difference between the final and the initial manifold indexes, can be implemented by means of independent voltage and current sources. In particular, it is shown that it is always possible to solve the considered control problem by introducing a unique source in the two-terminal element. Several examples are employed to show how the approach applies to different circuits and to illustrate how the dynamical properties of the controlled dynamics depend on the features of the designed voltage and current sources. 
Several future research issues can be foreseen, such as the extension to more general classes of circuits, the design of the pulse shape in order to reduce the transient toward the attractor contained into the final manifold, the practical issues concerning the circuital realization of the feedforward pulse programmed control inputs.

Funding Open access funding provided by Università degli Studi di Firenze within the CRUI-CARE Agreement. This work was supported by the project "Analogue COmputing with Dynamic Switching Memristor Oscillators: Theory, Devices and Applications (COSMO)," Grant PRIN 2017LSCR4K 002, funded by the Italian Ministry of Education, University and Research (MIUR).

Availability of data and material The datasets generated and analyzed during the current study are available from the corresponding author on reasonable request.

\section{Declarations}

Conflict of interest All authors certify that they have no affiliations with or involvement in any organization or entity with any financial interest or non-financial interest in the subject matter or materials discussed in this manuscript.

Open Access This article is licensed under a Creative Commons Attribution 4.0 International License, which permits use, sharing, adaptation, distribution and reproduction in any medium or format, as long as you give appropriate credit to the original author(s) and the source, provide a link to the Creative Commons licence, and indicate if changes were made. The images or other third party material in this article are included in the article's Creative Commons licence, unless indicated otherwise in a credit line to the material. If material is not included in the article's Creative Commons licence and your intended use is not permitted by statutory regulation or exceeds the permitted use, you will need to obtain permission directly from the copyright holder. To view a copy of this licence, visit http://creativecommons.org/licenses/ by/4.0/.

\section{Appendix}

Proof of Lemma 1 In the case of $A$ non-singular, it is enough to observe that (21) can be equivalently rewritten as

$$
\left\{\begin{array}{l}
v=-\lambda C A^{-1} \\
\mu=-\lambda C A^{-1} B .
\end{array}\right.
$$

If $A$ is singular, then $A$ has at least one eigenvalue equal to zero. Moreover, exploiting Assumption 1, it can be shown that while the algebraic multiplicity of the zero eigenvalue can be greater than one, the geo- metric multiplicity is exactly equal to one. This implies that the set of vectors $v^{\top} \in \mathbb{R}^{n}$ such that $v A=0$ is a one-dimensional linear subspace. Exploiting the observability PBH test (see, e.g., [57]), it can be also shown that the following relation:

$\operatorname{rank}\left(A^{\top} C^{\top}\right)=n$

holds. Hence, the proof follows by rewriting (21) in the equivalent form

$\left\{\begin{array}{l}A^{\top} v^{\top}=-\lambda C^{\top} \\ \mu \quad v B\end{array}\right.$

and observing that

$\operatorname{rank}\left(A^{\top}\right)<\operatorname{rank}\left(A^{\top} \lambda C^{\top}\right)$

for all $\lambda \neq 0$.

\section{References}

1. Bonomi, F., Milito, R., Zhu, J., Addepalli, S.: Fog computing and its role in the internet of things. In: Proceedings of the First Edition of the MCC Workshop on Mobile Cloud Computing. ACM, pp. 13-16 (2012)

2. M. Satyanarayanan. The emergence of edge computing. Computer, 50(1), 30-39, 2017

3. Williams, R.S.: What's next? [The end of Moore's law]. Comput. Sci. Eng. 19(2), 7-13 (2017)

4. Waldrop, M.M.: The chips are down for Moore's law. Nat. News 530(7589), 144 (2016)

5. Zidan, M.A., Strachan, J.P., Lu, W.D.: The future of electronics based on memristive systems. Nature Electronics 1(1), 22 (2018)

6. Krestinskaya, O., James, A.P., Chua, L.O.: Neuromemristive circuits for edge computing: a review. IEEE Trans. Neural Netw. Learn. Syst. 2019, 1-20 (2019)

7. Di Ventra, M., Pershin, Y.V., Chua, L.O.: Circuit elements with memory: Memristors, memcapacitors, and meminductors. Proceedings of the IEEE 97(10), 1717-1724 (2009)

8. F. Yuan, G. Wang, P. Jin, X. Wang, and G. Ma. Chaos in a meminductor-based circuit. Int. J. Bifurcat. Chaos, 26(08):1650130, 2016

9. Yuan, F., Wang, G., Shen, Y., Wang, X.: Coexisting attractors in a memcapacitor-based chaotic oscillator. Nonlinear Dynamics 86(1), 37-50 (2016)

10. Ielmini, D., Wong, H.S.P.: In-memory computing with resistive switching devices. Nature Electronics 1(6), 333-343 (2018)

11. Yang, J.J., Strukov, D.B., Stewart, D.R.: Memristive devices for computing. Nature nanotechnology 8(1), 13-24 (2013) 
12. Wang, Z., Ambrogio, S., Balatti, S., Ielmini, D.: A 2transistor/1-resistor artificial synapse capable of communication and stochastic learning in neuromorphic systems. Frontiers in neuroscience 8, 438 (2015)

13. Kumar, S., Strachan, J.P., Williams, R.S.: Chaotic dynamics in nanoscale $\mathrm{NbO} 2$ Mott memristors for analogue computing. Nature (2017)

14. Ielmini, D., Pedretti, G.: Device and circuit architectures for in-memory computing. Adv. Intell. Syst. 2000040 (2020)

15. Ascoli, A., Tetzlaff, R., Kang, S.M., Chua, L.O.: Theoretical foundations of memristor cellular nonlinear networks: A $\mathrm{DRM}_{2}$-based method to design memcomputers with dynamic memristors. IEEE Trans. Circuits Syst. I: Reg. Pap. 67(8), 2753-2766 (2020)

16. Ascoli, A., Messaris, I., Tetzlaff, R., Chua, L.O.: Theoretical foundations of memristor cellular nonlinear networks: Stability analysis with dynamic memristors. IEEE Trans. Circuits Syst. I: Reg. Pap. 67(4), 1389-1401 (2020)

17. Itoh, M., Chua, L.O.: Memristor oscillators. Int. J. Bifurcat. Chaos 18(11), 3183-3206 (2008)

18. Corinto, F., Ascoli, A., Gilli, M.: Nonlinear dynamics of memristor oscillators. IEEE Trans. Circuits Syst. I, Reg. Papers 58(6), 1323-1336 (2011)

19. B. Bao, Z. Ma, J. Xu, Z. Liu, and Q. Xu. A simple memristor chaotic circuit with complex dynamics. Int. J. Bifurcat. Chaos, 21(9), 2629-2645, 2011

20. L. O. Chua. Resistance switching memories are memristors. Appl. Phys. A, 102:765-783, 2011

21. Riaza, R.: Manifolds of equilibria and bifurcations without parameters in memristive circuits. SIAM J. Applied Mathematics 72(3), 877-896 (2012)

22. A. Amador, E. Freire, E. Ponce, and J. Ros. On discontinuous piecewise linear models for memristor oscillators. Int. J. Bifurcat. Chaos, 27(06):1730022, 2017

23. E. Ponce, J. Ros, E. Freire, and A. Amador. Unravelling the dynamical richness of 3D canonical memristor oscillators. Microelectron. Eng., 182:15-24, 2017

24. Corinto, F., Forti, M.: Memristor circuits: Flux-charge analysis method. IEEE Trans. Circuits Syst. I, Reg. Papers 63(1), 1997-2009 (2016)

25. Corinto, F., Forti, M.: Memristor circuits: Bifurcations without parameters. IEEE Trans. Circuits Syst. I, Reg. Papers 64(6), 1540-1551 (2017)

26. Chen, M., Sun, M., Bao, B., Wu, H., Xu, Q., Wang, J.: Controlling extreme multistability of memristor emulator-based dynamical circuit in flux-charge domain. Nonlinear Dynamics 91(2), 1395-1412 (2018)

27. Bao, H., Jiang, T., Chu, K., Chen, M., Xu, Q., Bao, B.: Memristor-based canonical Chua's circuit: Extreme multistability in voltage-current domain and its controllability in flux-charge domain. Complexity 2018 (2018)

28. M. Messias, C. Nespoli, and V. A. Botta. Hopf bifurcation from lines of equilibria without parameters in memristor oscillators. Int. J. Bifurcat. Chaos, 20(02), 437-450, 2010

29. Q. Li, S. Hu, S. Tang, and G. Zeng. Hyperchaos and horseshoe in a 4D memristive system with a line of equilibria and its implementation. Int. J. Circuit Theory Appl., 42(11):1172-1188, 2014

30. M.C. Scarabello and M. Messias. Bifurcations leading to nonlinear oscillations in a $3 \mathrm{D}$ piecewise linear memristor oscillator. Int. J. Bifurcat. Chaos, 24(1):1430001, 2014
31. Bao, B., Jiang, T., Xu, Q., Chen, M., Wu, H., Hu, Y.: Coexisting infinitely many attractors in active band-pass filter-based memristive circuit. Nonlinear Dynamics 86(3), 1711-1723 (2016)

32. Bao, B., Jiang, T., Wang, G., Jin, P., Bao, H., Chen, M.: Twomemristor-based Chua's hyperchaotic circuit with plane equilibrium and its extreme multistability. Nonlinear Dyn. 2017, 1-15 (2017)

33. Zhang, J., Liao, X.: Effects of initial conditions on the synchronization of the coupled memristor neural circuits. Nonlinear Dynamics 95(2), 1269-1282 (2018)

34. Chen, C., Chen, J., Bao, H., Chen, M., Bao, B.: Coexisting multi-stable patterns in memristor synapse-coupled Hopfield neural network with two neurons. Nonlinear Dynamics 95(4), 3385-3399 (2019)

35. H. Chang, Y. Li, F. Yuan, and G. Chen. Extreme multistability with hidden attractors in a simplest memristor-based circuit. Int. J. Bifurcat. Chaos, 29(06):1950086, 2019

36. Corinto, F., Forti, M.: Memristor circuits: Pulse programming via invariant manifolds. IEEE Trans. Circuits Syst. I, Reg. Papers 65(4), 1327-1339 (2018)

37. Chen, M., Sun, M., Bao, H., Hu, Y., Bao, B.: Flux-charge analysis of two-memristor-based Chua's circuit: Dimensionality decreasing model for detecting extreme multistability. IEEE Trans. Ind. Electron. 67(3), 2197-2206 (2020)

38. Karthikeyan, R., Jafari, S., Karthikeyan, A., Srinivasan, A., Ayele, B.: Hyperchaotic memcapacitor oscillator with infinite equilibria and coexisting attractors. Circuits, Systems, and Signal Processing 37(9), 3702-3724 (2018)

39. Yuan, F., Deng, Y., Li, Y., Wang, G.: The amplitude, frequency and parameter space boosting in a memristormeminductor-based circuit. Nonlinear Dynamics 96(1), 389-405 (2019)

40. Corinto, F., Di Marco, M., Forti, M., Chua, L.O.: Nonlinear networks with mem-elements: Complex dynamics via fluxcharge analysis method. IEEE Transactions on Cybernetics 50(11), 4758-4771 (2020)

41. Ma, X., Mou, J., Liu, J., Ma, C., Yang, F., Zhao, X.: A novel simple chaotic circuit based on memristor-memcapacitor. Nonlinear Dynamics 100(3), 2859-2876 (2020)

42. Innocenti, G., Di Marco, M., Tesi, A., Forti, M.: Input-output characterization of the dynamical properties of circuits with a memelement. Int. J. Bifurcation Chaos 30(07), 2050110 (2020)

43. D. P. Atherton. Nonlinear Control Engineering. Van Nostrand Reinhold, London, 1975

44. A. I. Mees. Dynamics of Feedback Systems. Wiley, New York, 1981

45. Khalil, H.K.: Nonlinear systems, 3rd edn. Prentice-Hall, Upple Saddle River, New Jersey (2002)

46. Di Marco, M., Forti, M., Innocenti, G., Tesi, A.: Harmonic balance method to analyze bifurcations in memristor oscillatory circuits. International Journal of Circuit Theory and Applications 46(1), 66-83 (2018)

47. Innocenti, G., Di Marco, M., Forti, M., Tesi, A.: Prediction of period doubling bifurcations in harmonically forced memristor circuits. Nonlinear Dynamics 96(2), 1169-1190 (2019)

48. Pisarchik, A.N., Feudel, U.: Control of multistability. Physics Reports 540, 167-218 (2014) 
49. Appeltant, L., Soriano, M.C., Van der Sande, G., Danckaert, J., Massar, S., Dambre, J., Schrauwen, B., Mirasso, C.R., Fischer, I.: Information processing using a single dynamical node as complex system. Nature Communications 2, 468 (2011)

50. Jensen, J.H., Tufte, G.: Reservoir computing with a chaotic circuit. In: European Conference on Artificial Life 14, 14:222-229. MIT Press (2017)

51. Di Marco, M., Forti, M., Corinto, F., Chua, L.O.: Unfolding nonlinear dynamics in analogue systems with memelements. IEEE Transactions on Circuits and Systems I: Regular Papers 68(1), 14-24 (2021)

52. M. Di Marco, G. Innocenti, M. Forti, and A. Tesi. Control design for targeting dynamics of memristor MuraliLakshmanan-Chua circuit. In 18th European Control Conference (ECC2019), 4332-4337, 2019

53. Ahamed, A.I., Lakshmanan, M.: Discontinuity induced Hopf and Neimark-Sacker bifurcations in a memristive Murali-Lakshmanan-Chua circuit. Int. J. Bifurcat. Chaos 27(06), 1730021 (2017)

54. Di Marco, M., Forti, M., Innocenti, G., Tesi, A., Corinto, F.: Targeting multistable dynamics in a second-order memristor circuit. In: 2020 IEEE International Symposium on Circuits and Systems (ISCAS), pp. 1-5 (2020)
55. Di Marco, M., Forti, M., Innocenti, G., Tesi, A.: Input design for controlling dynamics in a second-order memristive circuit. In: 2020 European Conference on Circuit Theory and Design (ECCTD), pp. 1-4 (2020)

56. Chua, L.O.: Introduction to memristors. IEEE Digital Library: https://ieeexplore.ieee.org/courses/details/ EDP091, 2009 (reaffirmed 2013)

57. Sontag, E.D.: Mathematical Control Theory: Deterministic Finite Dimensional Systems, vol. 6. Springer Science \& Business Media (1998)

58. Hautus, M.L.J.: Strong detectability and observers. Linear Algebra and its Applications 50, 353-368 (1983)

59. Horn, P., Moschytz, G.: Active RC single-opamp design of driving-point impedances. IEEE Transactions on Circuits and Systems 26(1), 22-30 (1979)

Publisher's Note Springer Nature remains neutral with regard to jurisdictional claims in published maps and institutional affiliations. 